\title{
RESEARCH
}

Open Access

\section{Antibiotics create a shift from mutualism to competition in human gut communities with a longer-lasting impact on fungi than bacteria}

Bastian Seelbinder ${ }^{1 \dagger}$, Jiarui Chen ${ }^{1,2+}$, Sascha Brunke ${ }^{3}$, Ruben Vazquez-Uribe ${ }^{4}$, Rakesh Santhaman ${ }^{1}$, Anne-Christin Meyer ${ }^{3}$, Felipe Senne de Oliveira Lino ${ }^{4}$, Ka-Fai Chan ${ }^{5}$, Daniel Loos ${ }^{1}$, Lejla Imamovic ${ }^{4}$, Chi-Ching Tsang ${ }^{5}$, Rex Pui-kin Lam ${ }^{6}$, Siddharth Sridhar ${ }^{5,7,8}$, Kang Kang ${ }^{1}$, Bernhard Hube ${ }^{4}$, Patrick Chiu-yat Woo ${ }^{5,7,8}$, Morten Otto Alexander Sommer ${ }^{4^{*}}$ and Gianni Panagiotou ${ }^{1,2^{*}}$ (D)

\begin{abstract}
Background: Antibiotic treatment has a well-established detrimental effect on the gut bacterial composition, but effects on the fungal community are less clear. Bacteria in the lumen of the gastrointestinal tract may limit fungal colonization and invasion. Antibiotic drugs targeting bacteria are therefore seen as an important risk factor for fungal infections and induced allergies. However, antibiotic effects on gut bacterial-fungal interactions, including disruption and resilience of fungal community compositions, were not investigated in humans. We analysed stool samples collected from 14 healthy human participants over 3 months following a 6-day antibiotic administration. We integrated data from shotgun metagenomics, metatranscriptomics, metabolomics, and fungal ITS2 sequencing.

Results: While the bacterial community recovered mostly over 3 months post treatment, the fungal community was shifted from mutualism at baseline to competition. Half of the bacterial-fungal interactions present before drug intervention had disappeared 3 months later. During treatment, fungal abundances were associated with the expression of bacterial genes with functions for cell growth and repair. By extending the metagenomic species approach, we revealed bacterial strains inhibiting the opportunistic fungal pathogen Candida albicans. We demonstrated in vitro how C. albicans pathogenicity and host cell damage might be controlled naturally in the human gut by bacterial metabolites such as propionate or 5-dodecenoate.
\end{abstract}

Conclusions: We demonstrated that antibacterial drugs have long-term influence on the human gut mycobiome. While bacterial communities recovered mostly 30-days post antibacterial treatment, the fungal community was shifted from mutualism towards competition.

\footnotetext{
* Correspondence: msom@bio.dtu.dk; Gianni.Panagiotou@hki-jena.de

${ }^{\dagger}$ Bastian Seelbinder and Jiarui Chen contributed equally to this work.

${ }^{4}$ Novo Nordisk Foundation Center for Biosustainability, Technical University of

Denmark, Kemitorvet 220, DK-2800 Lyngby, Denmark

'Leibniz Institute for Natural Product Research and Infection

Biology-Systems Biology and Bioinformatics, Hans Knöll Institute,

Adolf-Reichwein-Straße 23, 07745 Jena, Germany

Full list of author information is available at the end of the article
}

C C The Author(s). 2020 Open Access This article is licensed under a Creative Commons Attribution 4.0 International License, which permits use, sharing, adaptation, distribution and reproduction in any medium or format, as long as you give appropriate credit to the original author(s) and the source, provide a link to the Creative Commons licence, and indicate if changes were made. The images or other third party material in this article are included in the article's Creative Commons licence, unless indicated otherwise in a credit line to the material. If material is not included in the article's Creative Commons licence and your intended use is not permitted by statutory regulation or exceeds the permitted use, you will need to obtain permission directly from the copyright holder. To view a copy of this licence, visit http://creativecommons.org/licenses/by/4.0/ The Creative Commons Public Domain Dedication waiver (http://creativecommons.org/publicdomain/zero/1.0/) applies to the data made available in this article, unless otherwise stated in a credit line to the data. 


\section{Background}

The human gut microbiome is a complex ecosystem of bacteria, fungi, archaea, and phages [1]. The majority of research has focused on the bacterial part of the gut microbiome and their role in health and disease [2-4]. However, the critical role of fungi in host homeostasis remains is less well studied. Fungal dysbiosis may increase symptoms of inflammation, especially in the gut lumen [5]. Treating mice with fluconazole, an antifungal drug, increases the immune response and severity of experimentally induced colitis [6] but also induced allergic airway disease [7]. Fluconazole seems to substantially impact only certain types of fungi such as Candida, but not Aspergillus species [6].

Antibiotic treatment has a well-established detrimental effect on the composition of gut bacteria [8-11], but the effect on the fungal community is less clear. Nevertheless, antibiotic use is linked to overgrowth of particular fungi at multiple body sites $[7,8,12]$. Noverr et al. used a murine model to induce development of airway allergies by enriching for Candida and Aspergillus species in the gut followed by antibiotic treatment [10]. Theoretically, commensal bacteria may limit fungal colonization by production of antifungal compounds [13], competition for available nutrients, cellular contact, chemotaxis, or physiochemical changes to the local environment [14, 15]. Fungi defend themselves by secreting molecules, forming biofilms or forming mutualistic bonds with other bacteria. Candida albicans, for example, secretes the metabolite farnesol which interferes with the quorum-sensing of Pseudomonas aeruginosa [14, 15]. However, C. albicans can also enhance biofilm formation by Staphylococcus aureus in vitro. Pseudomonas fluorescens promotes the growth of the mycorrhizal fungus Laccaria bicolor in soil. Which bacterial-fungal interactions take place in the gastrointestinal tract of humans remains to be investigated. To date, the complex community of gut microbes is thought to be coevolved to maintain relative homeostasis in healthy humans [16].

Defining gut fungal consortia and their stability, resilience, and dynamics may reveal cause-effect relationships with bacteria. Although evidence is available on bacterial-fungal interactions in the gut at the taxonomic level [13-15], we do not have a comprehensive understanding of how bacterial functions influence the growth of particular fungi. Bacterial microbiome studies were often performed by amplifying the DNA of the ribosomal 16S gene. However, metagenome shotgun sequencing allows species- and sometimes even strain-level taxonomic classification, as well as the estimation of gene functions [17-19]. Furthermore, gene expression in microbial communities is not strictly matched with metagenomic potential [20]. Often, studies neglect the high transcriptional activity of some less-abundant species to metabolic functions.

In order to better understand the microbiome, we provide data to follow both, the bacterial and fungal communities of the lower human gastrointestinal tract over 3 months after antibiotic treatment concomitantly. We provide an overview of how the mycobiome and its interactions with the bacterial microbiome change and we reveal dependencies of specific fungal species from bacterial functions at DNA and RNA levels.

\section{Results \\ Antibiotic treatment triggers long-lasting dynamics at fungal species level}

We included 14 healthy human participants, 12 receiving the antibiotic intervention and 2 controls. Stool samples were collected at 4-6 time points per participants. We used 5 different antibiotics (one for each pair of treated participants). Samples were collected 15 days before administration of antibiotics (baseline), at 4 and 6 days of treatment (during treatment [DT]), 15 and 30 days after (early post treatment [EPT]), and 90 days after treatment (late post treatment [LPT]). We built high-quality libraries for ITS2 sequencing for 59 of 74 available stool samples. We estimated the fungal relative abundance using the PIPITS pipeline [21]. ITS sequences were clustered into operational taxonomic units (OTU) and taxonomically annotated using Mothur [22]. Antibiotic treatment led to a significant increase in species-level fungal alpha diversity during early post treatment compared to baseline (Fig. 1a; two-sided Wilcoxon rank-sum test, $p=$ 0.016, $q=0.094)$. Controls showed a considerable increase as well, although statistical significance could not be estimated due to the number of subjects $(n=2)$. At the level of individual antibiotic drugs (Suppl. Fig. 1), Augmentin and ciprofloxacin more than doubled baseline diversity. In contrast, changes for doxycycline and azithromycin were mild. Beta diversity using Bray-Curtis was not significantly different between time points in treated samples (Fig. 1b; PERMANOVA, $p>0.05$ ).

We subsequently investigated differences in fungal genera relative abundance over time. Candida genus increased 15-fold from baseline to treatment ( $q=0.004$; Suppl. Table 1). Candida increase was observed for all antibiotics except Augmentin (Suppl. Fig. 2). At the species level, results were more distinct (Suppl. Table 2) and for this analysis, we considered only prevalent fungal species (defined as present in $15 \%$ of samples). Comparing relative abundance changes from baseline to during treatment, only Saccharomyces sydowii decreased significantly $(q<0.05)$. However, the opportunistic pathogen Candida albicans tended to increase 7 -fold $(q<0.07)$ and was affected the most by Augmentin and doxycycline (Suppl. Fig. 3). Furthermore, C. albicans was 
a

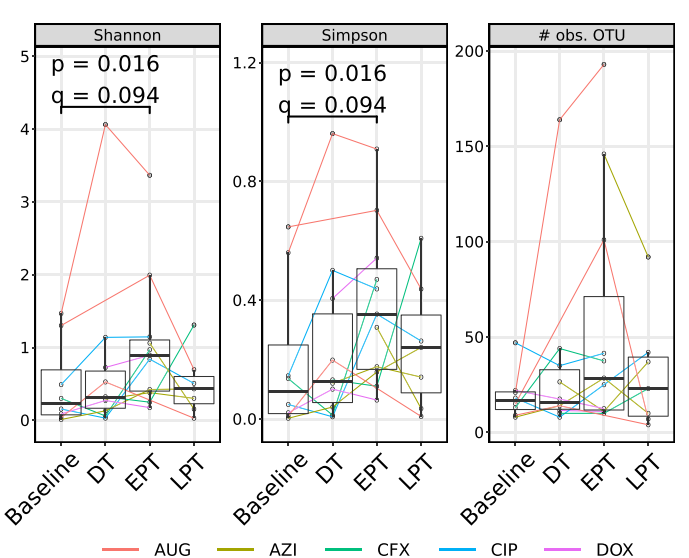

C
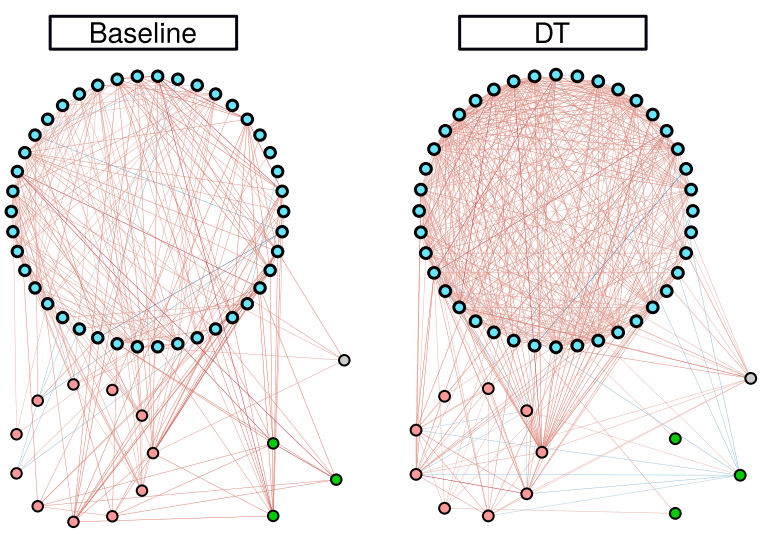

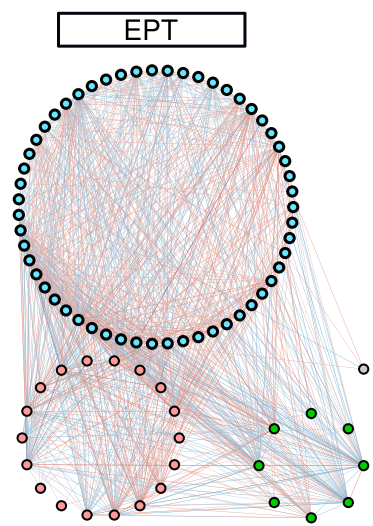

b

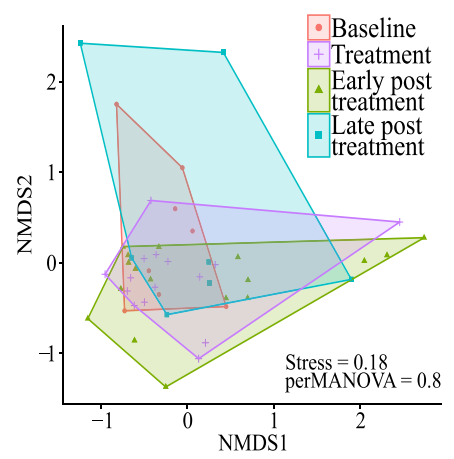

$\mathrm{d}$

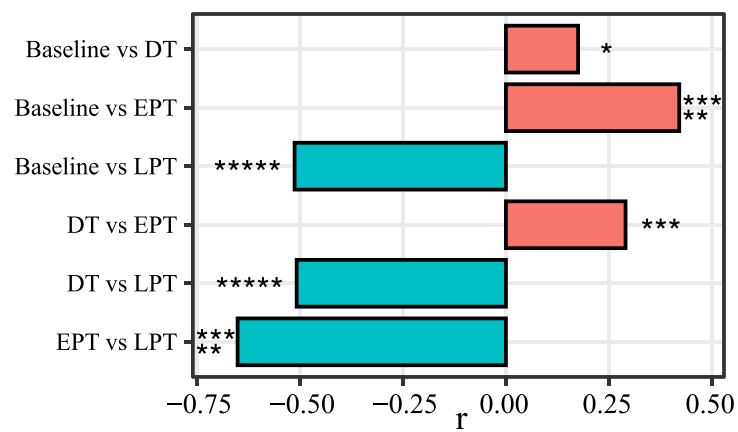

Fig. 1 Antibiotic treatment induces fungal competition. Statistical testing by Wilcoxon signed-rank tests with $p$ values adjusted for multiple testing using false discovery rate (FDR) ( $q=$ FDR[p]). Not significant, ns: $q \geq 0.05 ;{ }^{*} q<0.05 ;{ }^{* *} q<0.01$; ${ }^{* * *} q<1 \mathrm{e}-3$; ${ }^{* * * *} q<1 \mathrm{e}-4$; ${ }^{* * * * *} q<1 \mathrm{e}-5$. a, b Diversity analysis of samples from treated participants using PIPITS operational taxonomic units (OTU) relative abundances. a Boxplots showing Shannon (left) and Gini-Simpson indexes (middle) and species richness (right) with median (centerlines), first and third quartiles (box limits), and 1.5x interquartile range (whiskers). No significant changes were observed $(q<0.05)$. b Non-metric dimensional scaling of Bray-Curtis distance as a measure of beta diversity. No significant differences $(p<0.05)$ were found between time points using PERMANOVA. $\mathbf{c}$, $\mathbf{d}$ Co-abundance network analysis using BAnOCC. Only OTUs with prevalence $10 \%$ and significant correlations ( $95 \%$ credibility interval) with $|r| \geq 0.3$ were used for network construction. Networks were created independently for baseline, during (DT), early post (EPT), and late post treatment (LPT) to show temporal changes in fungal communities. c Fungal networks. Node colour indicates fungal phyla. Blue, Ascomycota; red, Basidiomycota; green, Mucoromycotina; grey, unknown. Edge colour indicates correlation type. Red, positive; blue, negative. $\mathbf{d}$ Network properties. Bar plots show number of nodes that increased and decreased in node degree centrality

detected in nine participants after treatment even though in only five at baseline.

Twenty-three species changed significantly from treatment to early post treatment periods $(q<0.05)$, many of which were not present before or after treatment. Many common fungi like Saccharomyces spp., opportunistic pathogens such as C. albicans, C. parapsilosis, and Malassezia restricta-a fungus recently connected to 
pancreatic cancer [23]-decreased in abundance, whereas less common fungi such as Candida boidinii increased in abundance. A minor decrease in abundance of C. albicans was also observed in controls, but not nearly as much.

To test for long-lasting changes, we compared relative abundances at baseline to late post treatment and found six species with significant changes. We further noticed that only 14 fungal species passed the prevalence filter at baseline and late post treatment, whereas up to 44 were observed during and early post treatment, suggesting that antibiotics temporarily created a niche for less common fungal species. In summary, the number of detected, prevalent species more than doubled during treatment and early post treatment, but these species had not successfully colonized 3 months later. Most changes were found within the first month after treatment, implying a delayed response of the fungal community to the treatment. Over one third of the fungal species present before treatment showed significant changes even 90 days after treatment.

\section{Antibiotic treatment increases co-exclusion in fungal communities}

We evaluated changes induced in the mycobiome from antibiotic administration by creating co-abundance networks based on ITS abundances. Networks were created for baseline and for during, early, and late post treatment periods (Fig. 1d). Only significant edges (95\% credibility) with absolute correlation of at least 0.3 were retained. Generally, we found significant correlations within and between Ascomycota, Basidiomycota, and Mucoromycotina species. At baseline, we found mostly positive correlations (240 positive and 10 negative) among 57 fungal species. During treatment, the number of correlations almost doubled (406 positive, 17 negative), whereas at early post treatment, correlation numbers doubled again. In contrast to the previous networks, more than half of the significant correlations were negative (399 negative, 550 positive), implying a major switch from mutualistic relationships at baseline and during treatment to competition between fungal species as they try to re-establish a stable community. We also observed these negative correlation patterns within and between fungal phyla. At late post treatment, this conflict persisted. Most co-abundance patterns had disappeared-only 25 correlations among 15 fungal species remained. We confirmed these trends by testing for significant changes in node degree centrality (Fig. 1e; Suppl. Table 3).

In conclusion, based on diversity, abundance, and network analysis, we observed that gut fungal communities started to change alongside the bacterial communities during treatment. Many fungi failed to colonize successful and competition emerged during early post treatment, leading to changes that lasted 90 days after treatment. The human mycobiome became more stochastic, leading to strikingly less co-abundance patterns among fungal species. These findings indicated that the gut mycobiome was not resilient enough to recover from the influence of antibiotics within 3 months.

\section{Changes in functional metagenomic diversity from antibiotics are not strictly followed by changes in metatranscriptomic diversity}

We characterized the subjects' microbiomes at baseline (Suppl. Fig. 4) and found that bacterial communities were dominated by bacteria from the Bacteroidetes and Firmicutes phyla but with strong variation in ratio, as expected in healthy individuals $[16,24]$. In line with previous studies, we observed a significant decrease in bacterial species alpha diversity (Suppl. Fig. 5). Ciprofloxacin had the strongest $(-40 \%)$ and cefuroxime the weakest negative effect $(-5 \%)$, whereas controls only an insignificant increase (2\%) (Suppl. Fig. 6). Beta diversity was significantly different during antibiotic treatment, but not in controls. In addition, we found that antibiotic treatment had the strongest influence on moderately abundant bacterial species (Suppl. Fig 5). We then estimated bacterial growth using GRiD [25] (Suppl. Table 4). In antibiotic-treated subjects, median growth of species decreased significantly during treatment compared to baseline as expected ( $p=0.009, r=-0.56$, Suppl. Fig. $7)$. Interestingly, the number of species with growth rate greater 1 increased significantly ( $p=0.0016, r=0.68$ ).

We subsequently investigated functional changes based on bacterial gene family abundance in the metatranscriptome and the metagenome. Alpha diversity of relative DNA gene family abundance was significantly reduced during treatment compared to baseline (Fig. 2a; $q<0.05$ ), but not in controls (Suppl. Fig. 8). Despite the changes at the DNA level, the alpha diversity for relative RNA gene family abundance did not change significantly between time points (Fig. 2b; $q>0.05$ ).

We investigated differences in beta diversity of gene family abundances based on Bray-Curtis dissimilarity (Fig. 2c, d). We performed ordination and statistical testing using distance-based redundancy analysis (dbRDA) using "subject id" as a constrained variable and "sample time" as an independent variable. In treated subjects, both DNA and RNA functional abundances showed significant differences in centroids between timepoints (Fig. 2c, d; DNA: $F$ $=2, p=0.002$, Df $=5$; RNA: $F=1.6, p=0.037$, Df $=5$ ). This was not observed in controls (Suppl. Fig. 9). Pairwise dbRDA revealed a significant difference from baseline to treatment in DNA functional abundance $(F=3.13, q=$ 0.014, Df $=1$; Suppl. Table 5). For RNA abundance, we observed only a trend $(F=1.6, p=0.085, \mathrm{Df}=1)$. Overall, 
a

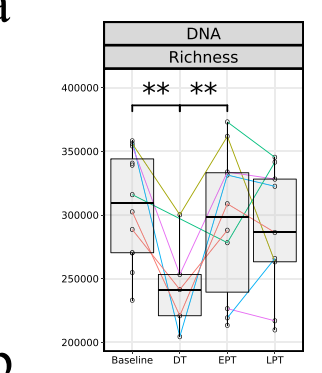

b
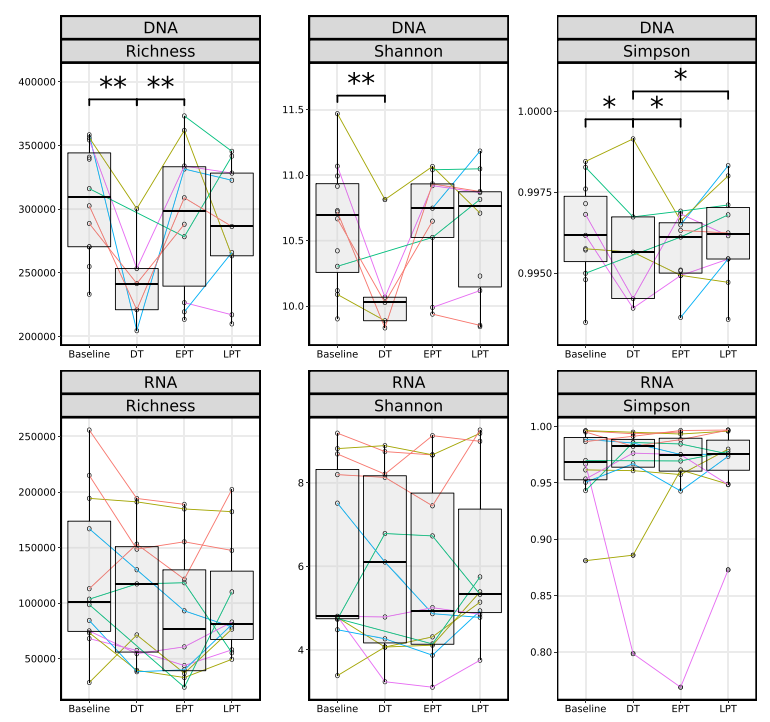

e

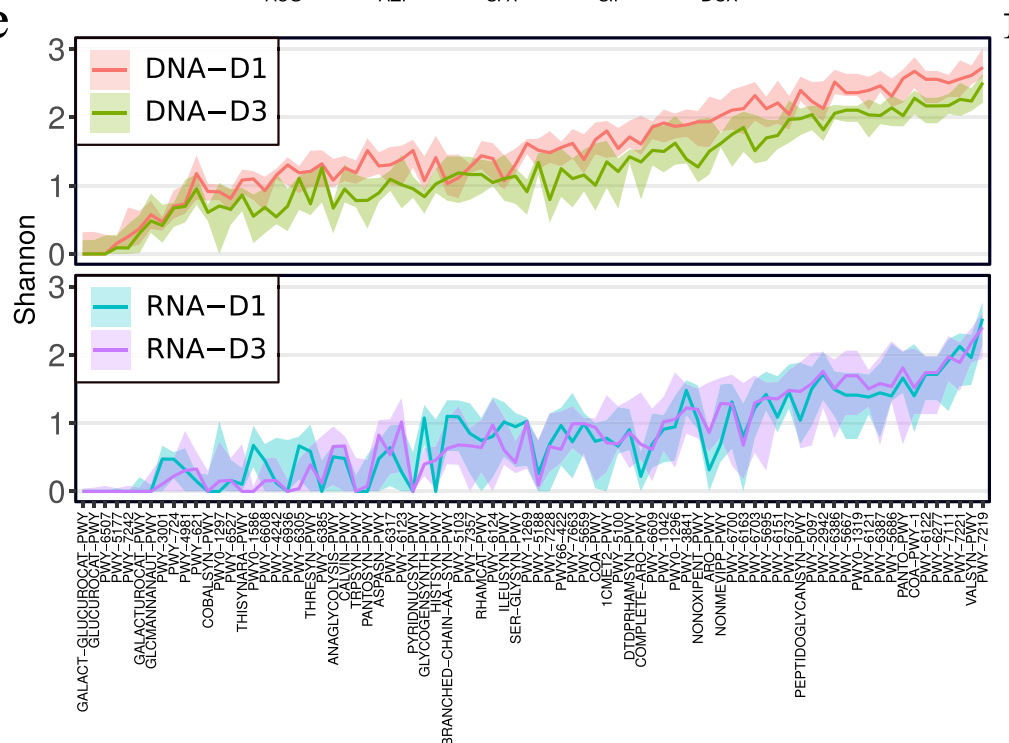

c
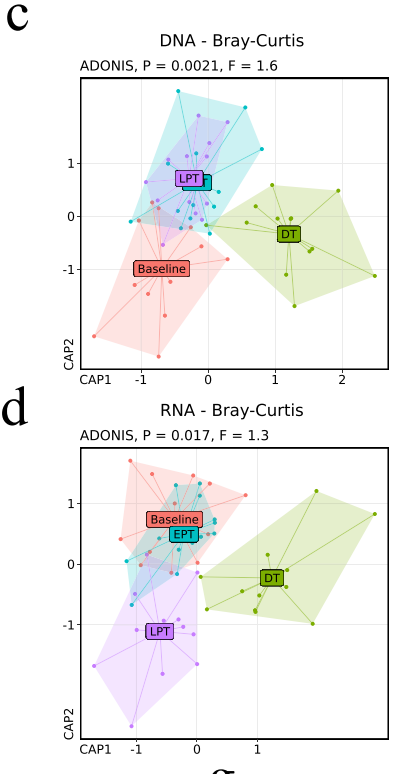

$\mathrm{f}$

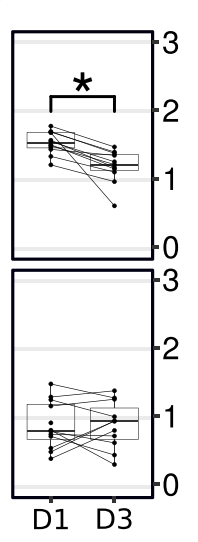

g

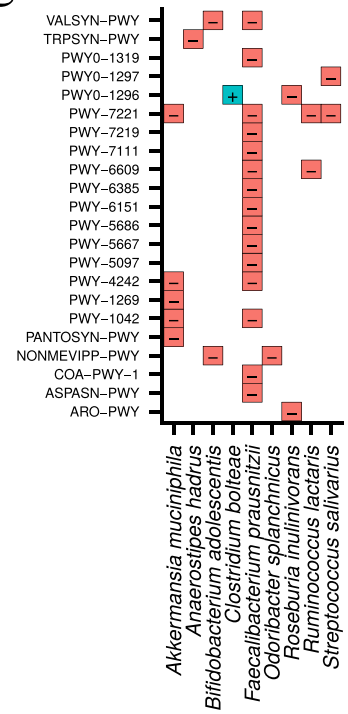

Fig. 2 Metagenomic contributional alpha diversity of metabolic function is severely reduced by antibiotic treatment. Diversity analysis of metagenomic and metatranscriptomic samples from participants using HUMAnN2 relative abundances. $\mathbf{a}, \mathbf{b}$ Alpha diversity of gene family relative abundances using a metagenomic and $\mathbf{b}$ metatranscriptomic data. Boxplots show species richness (left), Shannon (middle), and Gini-Simpson indexes (right) at 15 days before treatment (baseline), during (DT), and 30 days (EPT) and 90 days post treatment (LPT). Median (centerlines), first and third quartiles (box limits), and 1.5x interquartile range (whiskers) are shown. Lines between boxes connect same-donor samples. Statistical testing was by Wilcoxon signed-rank test with $p$ values adjusted for multiple testing using false discovery rate $\left(q ;{ }^{*} 0.01 \leq p<0.05\right)$. $\mathbf{c}$, $\mathbf{d}$ Constrained ordination of Bray-Curtis dissimilarity based on gene family abundances measured using principle coordinate analysis (PCOA). We used distance-based redundancy analysis to show the explained variance by sample time points while accounting for participant-specific influence. $\mathbf{e}-\mathbf{g}$ Contributional Shannon diversity of MetaCyc pathways of baseline and treatment samples. e Top, metagenomic and bottom, metatranscriptomic contributions. Mean (solid lines) and first and third quartiles (transparent ribbons) are shown. $\mathbf{f}$ Mean contributional diversity per participant per time point for DNA (top) and RNA (bottom). $\mathbf{g}$ Species with significantly increased (blue, + ) and decreased (red, -$)$ contribution to pathways based on two-sided Wilcoxon signed-rank test adjusted for compositionality $(q<0.1)$

these findings implied that the genetic potential of the bacterial community was reduced during treatment as expected. However, gene expression changes were considerably less compared to the metagenome and not as consistent among participants. Similarly, antibiotic treatment had no significant effect on the transcriptional activity of the core and variable metabolic pathways (as defined in [20]; Suppl. Fig. 10). In agreement with previous findings [20], the metatranscriptome was much more dynamic than the metagenome. 
Diversity of bacterial contribution to metabolic pathways is systematically reduced by antibiotics

We investigated if the contribution of species to a given pathway changed significantly over time [26] (Fig. 2g, h; Suppl. Fig. 11). By DNA, the median contributional alpha diversity of antibiotic-treated participants decreased significantly from baseline to treatment (Shannon: $\log _{2}$ fold-change [lf2] $=-0.4 ; q=0.015$; Simpson: lf2 $=-0.24 ; q=0.015)$. Controls showed no significant changes $(q>0.05)$. In contrast, we did not observe significant changes in alpha diversity measures for RNA ( $q$ $>0.05)$. We further investigated if the contribution of single bacterial species to metabolic pathways changed significantly between time points. We implemented a compositionality test as described in Palleja et al. [27], considering all pathways, and found 9 bacterial species whose contribution significantly changed $(q<0.1$; Fig. 2i). Important gut commensal bacteria including Akkermansia muciniphila, Faecalibacterium prausnitzii, Odoribacter splanchnicus, and Bifidobacterium adolescentis contributed less during treatment. A decline of such butyrate-producing species following antibiotic treatment has been observed before [11, 27]. In contrast, the multiantibiotic-resistant bacterium Clostridium bolteae [28] contributed more.

\section{Antibiotic treatment lastingly reduced bacterial-fungi interactions}

We increased the functional resolution of bacterial species using the metagenomic species (MGS) concept [29], which allows identification of taxonomically unidentified bacterial species. We further improved the method to identify some bacteria at the strain level based on their genetic potential. In contrast to previous studies, we used HUMAnN2 [19] gene family profiles as references in accordance with a published protocol [30]. HUMAnN2derived profiles allowed us to retrieve MGS with high purity (i.e. more than $95 \%$ of genes in an MGS group originated from the same species; Suppl. Table 6). We then identified 26 MGS with significant change in relative abundance during treatment compared to baseline (Suppl. Fig. 12; Suppl. Table 7), which was not observed in controls. Six of these had species-level annotation and were consistently decreased independent of the antibiotic drug used (Ruminococcus lactaris, Dialister invisus, Odoribacter splanchnicus, Bacteroidetes bacterium ph8, Akkermansia muciniphila, Bifidobacterium adolescentis; full list in Suppl. Figs. 13 and 14).

We combined MGS and ITS relative abundance data and used BAnOCC [31] to infer intra- and crosskingdom associations among bacterial and fungal species. We created co-abundance networks at the species level for baseline and for during, early post and late post treatment periods independently as described above
(Suppl. Fig. 15). In order to find significant changes in the structure of co-abundance networks, we compared differences in node degree. The degree of a node is defined by the number of significant correlations with that node. Hence, an increase in node degree implies an increase of potential interactions, i.e. an increase of potentially relevant effects. To study changes in bacterialfungal interactions, we tested for significant differences in node degree centrality considering only crosskingdom correlation (Fig. 3a, c; Suppl. Table 8). We observed a temporal increase in node degree during treatment compared to baseline $(q=0.055)$. From during to early post treatment, this degree dropped $(q=$ $0.0185)$ and decreased further at late post treatment $(q=$ 0.0185). To find lasting changes, we compared baseline against late post treatment and found significantly reduced degree $(q=0.00134)$. Considering these results in addition to the loss of correlations observed in the fungal network, we conclude that antibiotic treatment was a triggering event for disturbances in bacterial-fungal interactions. These disturbances ultimately drove gut bacteria and fungi towards independence.

We looked more closely at co-abundance patterns involving bacterial species with significant changes in abundance or pathway contribution during treatment (Fig. 3c; all significant correlation in Suppl. Table 9). C. bolteae increased in relative abundance during treatment and correlated positively with many fungal species during treatment, such as the opportunistic pathogen $C$. albicans, or the mycotoxin producers Aspergillus penicillioides and Penicillium glandicola. In contrast, $O$. splanchnicus was persistently negatively correlated with opportunistic pathogens from the genera Candida, Aspergillus, and Alterna. O. splanchnicus is part of the healthy gut community but rarely investigated in terms of its role. Roseburia inulinivorans was negatively associated to opportunistic pathogens C. albicans, C. sake, and P. glandicola. Low Roseburia abundance was associated with higher glucose levels and ulcerative colitis [32, 33]. Likewise, Eubacterium rectale was negatively associated with $C$. albicans and P. glandicola. Depending on the diet, Eubacterium rectale decreased glucose and insulin levels [34]. Notably, butyrate-producing species were negatively associated with at least one opportunistic fungal pathogen.

At last, we considered bacterial-fungal correlations together with MGS abundance changes during treatment and fungal abundance changes in early post treatment. Bacterial species with decreased relative abundance and negative correlation to a fungus that showed an increased abundance were considered competitors. For example, $O$. splanchnicus was decreased during treatment, and showed negative correlation to $C$. albicans. A list of possible bacterial-fungal competitors is shown in Suppl. Table 10. 


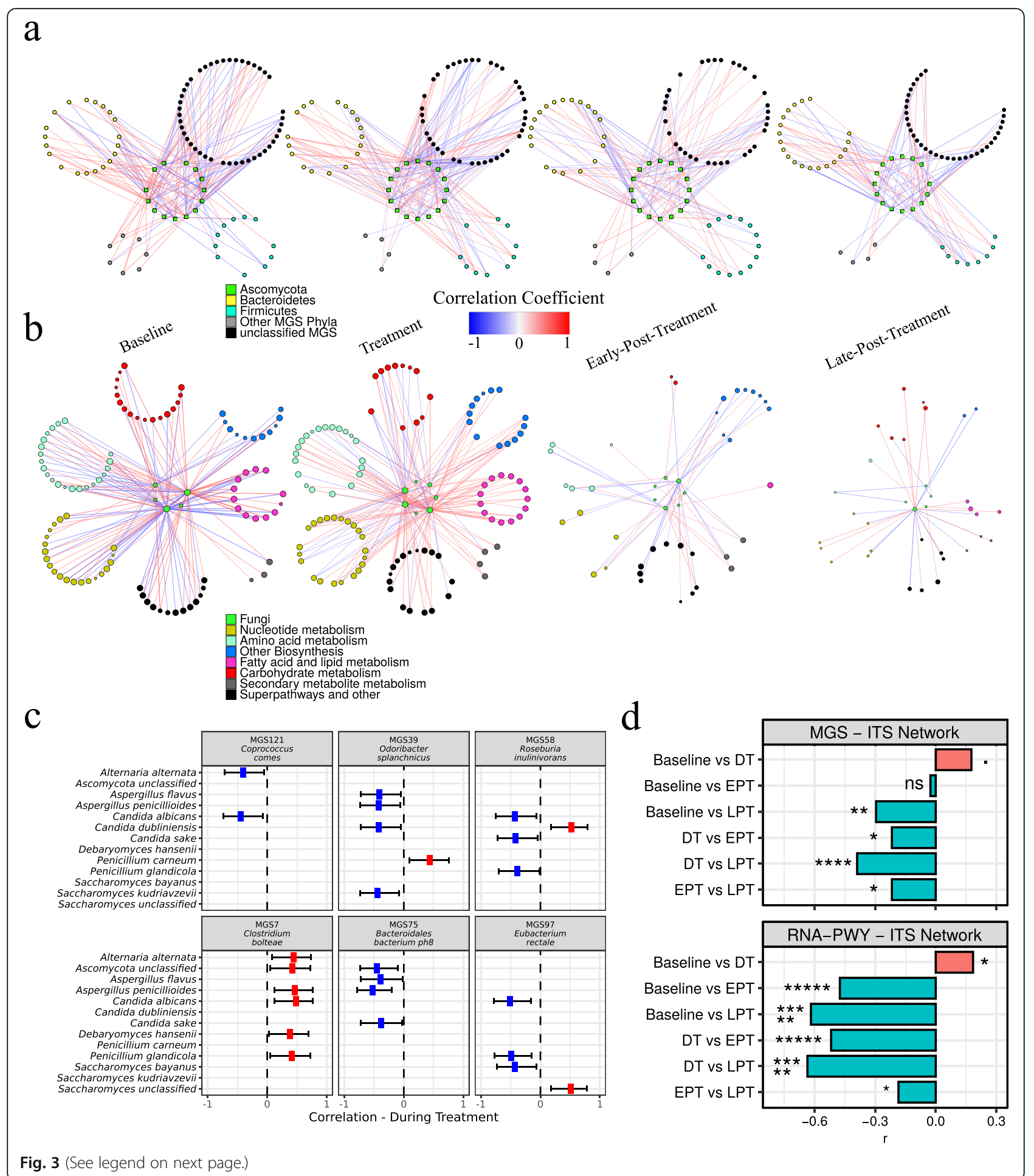


(See figure on previous page.)

Fig. 3 Cross-kingdom interactions among fungi, bacteria species, and pathway expression. $\mathbf{a}$, b Co-abundance networks at indicated time points using BAnOCC with a $25 \%$ and b $50 \%$ prevalence filter. Only significant edges are shown (based on $95 \%$ credibility interval) with $|r| \geq 0.3$.

Negative correlations (blue), positive correlations (red). Networks are left (baseline) to right (late post treatment). a Correlations among fungal and bacterial species based on metagenomic species (MGS) and internal transcribed spacer (ITS) relative abundance. b Correlations among fungal species and pathway expression based on HUMAnN2 RNA pathway and internal transcribed sequence relative abundance. Superpathways and other pathways that did not fit into the six major categories were grouped as "other". c Estimated correlation between bacterial and fungal species during treatment. Positive (red), negative (blue). Error lines show 95\% confidence intervals. $\mathbf{d}$ Effect size of node degree change. $r$ values change from -1 (100\% decrease) to 1 (100\% increase). (Top) MGS and ITS relative abundances. (Bottom) RNA-PWY and ITS relative abundances. Statistical testing for significant changes in node degree was performed using a two-sided Wilcox signed-rank test. $P$ values were adjusted for multiple testing using FDR. Node degree was determined independently for baseline, during (DT), early post (EPT), and late post treatment (LPT). Significance is indicated by symbols (ns, $q \geq 0.05 ;{ }^{*} q<0.05$; ${ }^{* *} q<0.01$; ${ }^{* * *} q<1 \mathrm{e}-3$; ${ }^{* * *} q<1 \mathrm{e}-4$; ${ }^{* * * *} q<1 \mathrm{e}-5$ )

\section{Prevalent fungi correlated with pathway expression during treatment}

We investigated relationships among metabolic pathway expression levels (MetaCyc database-PWY; metatranscriptomic abundance) and fungal ITS abundance (Fig. $3 \mathrm{~b})$ by creating co-abundance networks analogous to the bacteria-fungi network. We tested for significant changes in node degree considering only correlations between fungal OTUs and pathway expression. From baseline to treatment, node degree increased ( $q=0.0185$; Fig. 3d). Most correlations were positive (146 of 189) during treatment in contrast to baseline (82 of 153). Hence, and despite the increase of variance of metatranscriptome diversity during treatment, we still observed co-abundance with fungal species during treatment. This observation suggested a mutual influence between the fungal community and expression of bacterial metabolic pathways. About one third of correlations at baseline involved $C$. albicans and one third involved Saccharomyces. After treatment, node degree dropped significantly to below baseline levels (during vs. early post: $q=4 \mathrm{e}-11$; early vs. late post: $q=0.0185$; baseline vs. late post: $q=3 \mathrm{e}-15$ ). Almost all C. albicans co-abundance patterns were lost at 90 days post treatment, with Saccharomyces genus accounting for over $70 \%$ of remaining correlations (24 of 31). Overall, Saccharomyces appears to be more resilient with respect to bacterial metabolic pathways expression than other prevalent fungi.

We then increased our resolution by focusing on correlations between fungal OTU abundance and pathways in broader functional categories (Suppl. Fig. 16; Suppl. Table 11). We observed a significant increase in node degree from baseline to treatment for pathway functions in nucleotide metabolism $(q=$ 0.026 ) and biosynthetic pathways (e.g. for vitamins, tetrapyrroles, NAD) $(q=0.043)$. Almost all correlations were positive. We found no significant changes in remaining categories $(q<0.1$; metabolism of amino acids, carbohydrates, fatty acids and lipids, secondary metabolites). Node degree dropped significantly after treatment in all categories except secondary metabolite metabolism $(q<0.05)$.
Since our treatment targeted bacteria, we expected the bacterial community to respond to the selective pressure with strong, directed changes in pathway expression. Most metatranscriptomic changes appeared to be stochastic. Yet we still observed mostly positive co-abundance patterns between fungal abundance and bacterial pathway expression during treatment, especially with functions required for bacterial growth. Even though correlations do not imply causations, when performed on multiple different levels, they can still offer significant insights. The results suggested a common origin for changes in the mycobiome and pathway expression: if gut fungi generally take advantage of reduced complexity in the bacterial community, we would expect an increase in fungal diversity. However, we observed no systematic change. Highly abundant and adapted fungi may still overgrow. In both scenarios, we would expect an increase in negative correlations between fungal and bacterial abundances during treatment but found mostly positive correlations. Generally, antibiotics drove the mycobiome alongside the microbiome, leading to a temporal increase in fungal richness, but also increased fungal competition subsequently. On the long run, antibiotic treatment broke down most of the inferred relationships between bacteria and fungi, as shown by diverging mycobiomes 3 months after treatment.

\section{Key bacterial species and molecular mediators of Candida albicans colonization}

Our ITS data showed at least one C. albicans read per participant over 112 days but with varying relative abundance from 0 to $42 \%$. C. albicans was detected during treatment even if it was not detected at baseline, as in other studies [12,35], confirming antibiotic treatment as risk factor for colonization and overgrowth of this fungus. Furthermore, C. albicans significantly decreased 2 weeks after treatment, implying the indirect impact on its growth by the microbiome. We searched for metabolites detected in the human gut that may inhibit or promote $C$. albicans growth. We performed metabolomics analyses on a subset of 15 stool samples and calculated Spearman's correlations for the relative abundance of 
each metabolite and relative abundance of $C$. albicans by ITS (Suppl. Fig. 17). Based on these findings, we performed $C$. albicans growth assays in defined medium containing serial dilutions of selected metabolites. With several metabolites, including 4-hydroxybenzoic acid and 8,11,14-eicosatrienoic acid, we observed only minor growth reductions at the highest concentrations (Suppl. Fig. 18). More pronounced growth reduction occurred with adipic acid, aminoadipic acid, and ornithine, but fungi still grew with high concentrations of these metabolites. In contrast, propionic acid, acetic acid, and cis-5dodecenoic acid fully inhibited growth at a range of concentrations. We then tested if the substances directly damaged human cells. Using a human vaginal cell line (A431) without $C$. albicans, the bile acid lithocholate (LCA) and cis-5-dodecenoic acid showed limited cytotoxicity (Suppl. Fig. 19). No other substances caused detectable cell damage.

Next, we assayed the effect on human cells by C. albicans in presence of the same metabolites. At higher concentrations, when in vitro fungal growth was reduced, human cell damage decreased with the short-chain fatty acids (SCFAs) propionic $(p<0.05)$ and acetic acid $(p<$ $0.001)$. Acetic or cis-5-docenoic acid $(p<0.01)$ almost fully abolished cell damage by $C$. albicans. Benzoic acid reduced damage to a lesser extent $(p=0.051)$. Since the morphology (yeast or hyphal cells) is critical for its damaging potential, we investigated if the substances also led to morphological changes in C. albicans (Fig. 4e). On high concentrations, hyphae formation and growth were almost completely suppressed by 5 -dodecenoic and acetic acid. 5-dodecenoate also reduced hyphae formation under growth-permitting concentration. Glutathione only allowed formation of chains elongated yeasts resembling pseudohyphae. LCA partially suppressed hyphal growth at the high concentration, resulting in high numbers of pseudohyphae and yeast cells.

These metabolites that affect $C$. albicans growth negatively may also promote the growth of its fungal competitors, such as Saccharomyces spp., Penicillium spp. and Aspergillus spp. Therefore, we repeated the correlation analysis with the corresponding OTUs (Suppl. Table 12). For each fungal species, we found several metabolites with positive correlation. Considering metabolites negatively affecting C. albicans, only 2-methyl butanoic acid and 3hydroxy butyric acid were found to be significantly positively correlated with Penicillium spinulosum and LCA with Aspergillus flavus. Still, promotive effects on other fungal species need to be verified in future work.

\section{Bacterial supernatant inhibits C. albicans growth}

We investigated which gut bacteria might be the main direct or indirect producers or contributors of these compounds in our human participants. We correlated metabolite concentrations with MGS relative abundances (Suppl. Fig. 20) and focused on positive associations. We looked at species that correlated with multiple, different metabolites. Bacteroides coprophilus correlated with aminoadipic acid and acetate; C. comes with 4-hydroxybenzoic acid, 5-dodecenoate, and glutathione; F. prausnitzii with 4-hydroxybenzoic acid; $E$. lenta with 5-dodecenoate and eicosatrienoic acid; $B$. eggerthii with 5-dodecenoate and eicosatrienoic acid; and O. splanchnicus with acetate. All six species correlated with LCA or its derivates.

Our correlation methods helped us to pinpoint bacteria that may promote or inhibit C. albicans growth (Fig. 4a). For testing these predicted associations in vitro, we selected bacterial strains based on sufficient confidence in our strain-level inference in addition to significant correlation to C. albicans. We performed the strain identification directly from the MGS profiling. Instead of strain detection methods using single-nucleotide polymorphisms (e.g. StrainPhlAn [18], metaSNV [36], ConStrains [37]), we adopted a strategy based on gene content as in PanPhIAn [38]. We therefore analysed reads corresponding to a specific MGS. For example, gene coverage for O. splanchnicus strains for two participants (N, E; Fig. 4b; Suppl. Fig. 21) showed that both subjects had the highest coverage for strain DSM 20712, so we selected DSM 20712 for in vitro assays. In the end, we were interested in bacterial strains for which we found significant correlation with inhibitory metabolites, significant correlation with C. albicans, and high confidence from the strain inference. Based on these results, we selected Bacteroides eggerthii and Odoribacter splanchnicus for further in vitro experiments.

We determined the antifungal effect of metabolites produced by selected bacterial strains using their sterilized culture supernatants as growth medium for C. albicans. We measured C. albicans growth using $100 \%$ or $50 \%$ supernatant diluted in modified Gifu anaerobic media (mGAM) (Fig. 4c). Percentage inhibition was compared to optimal growth conditions in fresh medium. C. albicans growth was significantly inhibited by supernatants from $B$. eggerthii (50\% growth) or $O$. splanchnicus (40\%). Using 100\% bacterial supernatants had stronger effects, showing that inhibition was proportional to supernatant dilution (Suppl. Fig. 22). We tested two additional $C$. albicans strains to exclude that observed effects were strain specific but saw no differences (Suppl. Fig. 22). B. eggerthii and O. splanchnicus also inhibited $C$. albicans growth in pairwise in vitro coculturing experiments (Suppl. Fig. 23).

Finally, we analysed the supernatant of these species to characterize their metabolic capabilities that may relate to C. albicans growth (Suppl. Fig. 24-25). We included the supernatant from Ruminococcus [Blautia] torques as positive control, since this species was shown to have 


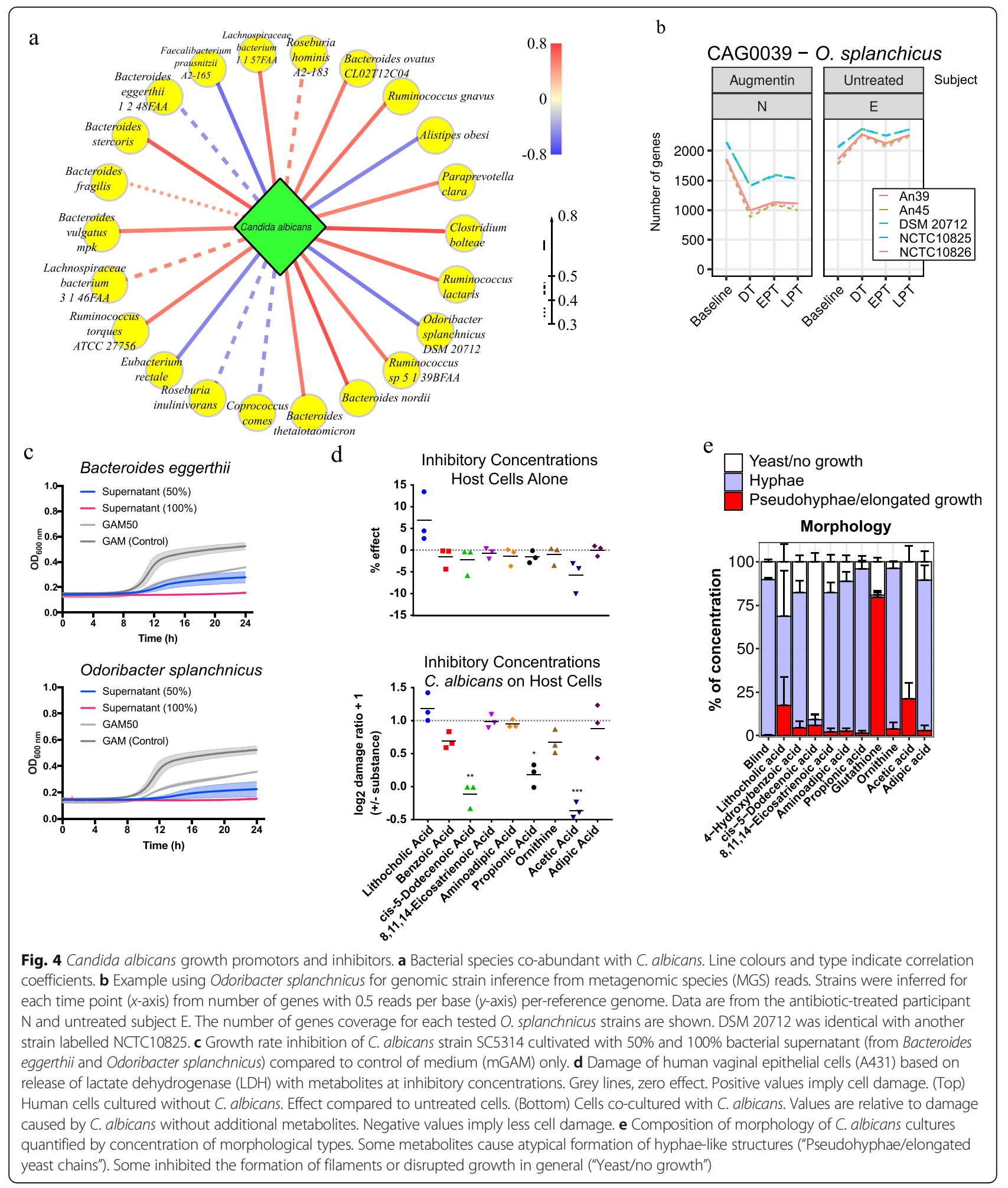

positive effect on C. albicans growth previously (Mirhakkak et al., 2020, under review) and correlated positively with $C$. albicans in our study. Compared to quality control samples, O. splanchnicus supernatant contained high concentrations of butyric acid (7-fold relative conc.), which suppresses C. albicans growth in vitro [39]. But we also measured elevated levels of the growth suppressing metabolites adipic and aminoadipic acid, and ornithine. In contrast, $B$. eggerthii supernatant contained elevated levels of acetic acid (1.6-fold), formic acid (3- 
fold), and hexanoic acid (2-fold). The full growthinhibiting metabolites 2-methyl-propanoic acid and propanoic acid were also found in supernatants of $O$. splanchnicus and B. eggerthii, but roughly 3-times higher in $B$. eggerthii. In contrast, $R$. torques produced only formic acid in higher abundance (1.25-fold), but almost none of the strong inhibitory SCFA. Altogether, the supernatant analysis shows that propionate, ornithine, and benzoic acid are effective inhibitors of C. albicans growth, and these compounds were likely produced by $B$. eggerthii and O. splanchnicus also in the human gut.

\section{Discussion}

Mouse models can offer some advantages for studying competitive relationships between gut bacteria and fungi. Previous studies have shown that antibiotics induce fungal overgrowth in the murine gut lumen $[12,35,40]$. However, antibiotic doses used in mice experiments create an almost germ-free environment after treatment, which is unlikely to apply to the human gut with clinical use of antibiotics. Furthermore, the mice gut microbiome and human gut microbiome differ considerably [41-43]. For example, many Firmicutes spp., which represent major colonizers of the human gut, cannot efficiently colonize the murine gut. Sovran et al. showed that Enterobacteriaceae play an important role for bacteria-fungi interactions in the murine gut [44]. In their study, Enterobacteriaceae accounted for 40 to $65 \%$ of reads in Vancomycin treated mice. We investigated the relative abundance of Enterobacteriaceae spp. in our human subjects. However, the accumulated relative abundance of Enterobacteriaceae spp. for most samples was below $1 \%$ before, during and after treatment (median 0.02\%; except for Augmentin with 13\%), making it difficult to assess whether Enterobacteriaceae were relevant for bacterial-fungal interactions in the human gut (Suppl. Fig. 26).

In this study, we investigated if fungal overgrowth was induced in the human gastrointestinal tract under physiological conditions. We present evidence that changes on fungal abundance at the species level are highly dynamic in the lower human gastrointestinal tract. Even though gut bacteria and fungi successfully prevented several temporarily detected fungi from colonization the lumen lastingly, we found significant alternations to the relative abundance of several fungi even 90 days after antibiotic treatment.

We used 5 different broad-spectrum antibiotics which are commonly used to treat human diseases [45]. Recent work by Maier et al. [46] addresses the issue that most knowledge of antibiotic drugs and their bacterial targets is based on pathogens and not the commensal microbiome. In a large screening of 144 different antibiotics and the 40 most common gut microbial strains, most antibiotics inhibited growth of all tested bacterial strains. Only Clostridium showed consistent resistance to many drugs. Indeed, some bacterial species are stronger or less affected depending on the antibiotic used. We investigated how much these expected differences apply to our data. Effect sizes varied, but overall, most of the significant changes were independent of specific antibiotic drugs. Because of our small cohort size, we cannot assess if the differences in effect sizes are due to differences in baseline communities or differential inhibition of the drugs. More work is required by using bigger cohorts as well as other antibiotic drugs with narrower targets.

Co-abundance networks inferred mutual relationships between fungal species at baseline and during treatment. Post treatment, however, competition emerged. Furthermore, we observed far fewer co-abundance patterns between fungi and bacteria in early and late post treatment periods, indicating profound decline in bacterial-fungal interactions. Overall, we found the fungal community to be less resilient than the bacterial. Based on these data, we hypothesize that the dominant gut fungi of healthy individuals were in balance with gut bacteria. Antibiotic administration induced profound changes to gut bacteria that translated into changes in fungal abundance that lasted until the end of our study period. Indeed, these results must be considered with caution, as we did not perform quantitative estimations of bacterial and fungal abundances. In most cases, relative abundance estimation does not allow inference of true direction of change [47]. For quantification, bacterial cells are counted by flow cytometry in addition to DNA sequencing or qPCR $[47,48]$. However, broad-spectrum antibiotics decrease bacterial cell counts by 3 orders of magnitude [47]. We estimated bacterial growth in situ to show that bacterial growth was significantly impaired at the community level. Hence, significant decrease in relative abundance of species will likely be reflected in true abundance as well. In future work, increasing the number of study subjects will help to increase certainty in and resolution of the findings.

One of the largest knowledge gaps about the basic biology of gut microbial balance is the lack of comprehensive functional analyses. Metatranscriptome studies have found both more [20] and less [49] uniformity in individual participants' profiles compared to respective metagenomes. Despite minor changes in beta diversity, we found no significant changes induced by antibiotic treatment in gene family alpha diversity, species contribution, or transcriptional activity of metabolic pathways. This result was most likely due to high variability in the metatranscriptome, as observed previously in healthy humans [20]. However, fungal abundance and bacterial growth may have influenced one another because mutual relations between fungal abundances and expression 
of bacterial functions for growth were inferred, especially during treatment. Because these patterns were not as pronounced before and after treatment, we identified antibiotic administration as the main driver of this change.

Understanding and finding microbial mediators of fungal pathogens may help to improve antifungal treatments. We focused our study on C. albicans, testing in vitro if growth was affected by compounds produced by two bacterial species, B. eggerthii and O. splanchnicus. Although the supernatant of each bacterium was used in combination with optimal C. albicans growth medium, the supernatants inhibited C. albicans growth considerably. Such a condition is plausible for the lower human intestine, because we expect most easily metabolizable compounds, e.g. carbohydrates, to be absorbed by the small intestine. Furthermore, the two species may be physically separated in the gut lumen. Some of the metabolites with clear growth reduction to $C$. albicans were found in bacterial supernatants. However, we cannot exclude potential promoting effects of other bacteria that could occur in the same vicinity.

A decline in gut bile acids and SCFA is linked to disease states [50,51], but cause-effect mechanisms are less understood. We identified five metabolites that naturally occur in the human gut to effectively inhibit growth and/or lower hyphae formation, a key attribute of $C$. albicans virulence [52]. Acetate is a prototypical SCFA that dampens the immune response at higher concentrations [53]. The SCFA propionate plays an important role in immune regulation [54]. Lithocholate is a secondary bile acid and such secondary bile acids may inhibit $C$. albicans growth [50]. Glutathione is an antioxidant that dampens cell damage [55]. Cis-5-dodecenoic acid suppressed hyphae formation entirely. A similar compound, cis-2-dodecenoic acid, is produced by Burkholderia cenocepacia and strongly interferes with $C$. albicans growth $[56,57]$. In contrast to previous studies $[39,51]$ we also show that acetate, 5-dodecenoic acid, and propionate also significantly reduced $C$. albicans-mediated host cell damage in vitro. These compounds could also support the growth of fungal C. albicans competitors. However, a correlation analysis between these metabolites and multiple different Saccharomyces, Penicillium, and Aspergillus spp. did not indicate that. Nevertheless, this needs to be experimentally verified in future work.

Several limitations should be highlighted. Observing gut bacterial and fungi concomitantly is difficult as long as bacterial and fungal abundances are estimated using two independent sequencing technologies. Improvements in correlation methods mitigate some of the resulting problems. Still, our correlation results regarding inter-kingdom species-species correlation could be improved in the future. Estimating cell counts per kingdom would further help to improve correlation estimates. Our findings are further limited to just the 5 antibiotic drugs used. Even though many significant findings seemed consistent across the drugs, increasing the number of patients for each drug would help to get more differentiated results. When studying mechanistic effects with respect to $C$. albicans growth, we could not simulate the complexity of the gut community. We aimed to find metabolic regulators, but the growth of fungi and bacteria in the gut is certainly based on a combination of several metabolic factors and environmental conditions. We looked at a variety of aspects from host cell damage to morphology, but these were still in vitro findings.

Our results indicated that antibiotic treatment has a longer-lasting impact on gut fungi than bacteria, driving fungal communities from mutualism to competition. This work also advanced MGS methods for resolving microbiome compositions and interactions. Of potential clinical relevance, we demonstrate how particular SCFAs and bile acids produced by gut bacteria restricted human cell damage from $C$. albicans but also show other compounds with considerable effects.

\section{Conclusions}

Theoretically, bacteria and fungi compete for resources available on the gut lumen, but they may also support one another. In this study, we investigated the temporal, concomitant changes of gut bacteria and fungi in humans. We demonstrate that antibacterial drugs have long-term influence on the human gut mycobiome, driving fungal communities from mutualism to competition. We further show how metabolites produced by bacteria such as cis-5dodecenoic acid may actively suppress pathogenicity of opportunistic fungi such as C. albicans. We thereby show that gut bacterial-fungal interactions are an important consideration for antibacterial treatment.

\section{Methods \\ Study design \\ Human participants}

Stool samples were gathered from 14 healthy adults, aged 18-65 years, from Denmark and Hong Kong. Samples were collected over 3-4 months. The Danish study was approved by the local ethics committee in Region Zealand, Denmark (SJ-383), and the Hong Kong study was approved by the Institutional Review Board of The University of Hong Kong/Hospital Authority Hong Kong West Cluster (UW 17-042). All work was performed in accordance with the Good Clinical Practice principles and the Helsinki Declaration. Written informed consent was obtained from all participants. Patient characteristics are described in (Suppl. Table 13). Subjects with any of the exclusion criteria below were not eligible for entry 
into the present study: (i) history of taking antibiotics over the last 6 months, (ii) receiving systemic antifungals/antifungal mouthwashes or probiotics concurrently, (iii) patients suffering from immunosuppressive conditions or taking immunosuppressants, and (iv) severe medical comorbidities requiring frequent hospitalization. Another cohort of six healthy, untreated individuals from Canada was acquired from a previous study from Raymond et al. [58].

\section{Treatment}

Of the participants, 12 were treated for 6 days with 1 antibiotic drug out of 5: doxycycline (tetracycline class), azithromycin (macrolide class), Augmentin ( $\beta$-lactam class), ciprofloxacin (quinolone class), and cefuroxime ( $\beta$-lactam class). Two untreated participants were used as controls.

\section{Sampling}

From each participant in the clinical study in Denmark, 6 stool samples were obtained: one 15 days before treatment ( \pm 1 day), two during treatment (days 3 and 5 of treatment \pm 1 day), and three at 15,30 , and 90 days after treatment ( \pm 1 day). From each participant in the clinical study in Hong Kong, four stool samples were obtained at 7 days before treatment ( \pm 1 day), day 6 of treatment, and 30 and 90 days after treatment. Collected samples were aliquoted and stored at $-80^{\circ}$ immediately after collection until DNA extraction. Stool samples of control patients treated with placebo [58] were acquired before, 7 days, and 90 days after treatment.

\section{Metagenomics and metatranscriptomics sequencing}

For participants in the clinical study in Denmark, bacterial metagenomics and metatranscriptomics raw data were obtained from Kang et al. (in preparation). Briefly, DNA was extracted using a MO BIO PowerMax Soil DNA Extraction Kit (MO BIO Laboratories, Inc) and purified with PowerClean Pro DNA Clean-Up Kits (MO BIO Laboratories, Inc.) according to the manufacturer's protocol. For RNA, rRNA was depleted using a RiboZero Gold rRNA removal kit-Epidemiology (Illumina). The remaining total RNA was extracted using a MO BIO PowerMicrobiome ${ }^{\text {Tm }}$ RNA Isolation Kit (MO BIO Laboratories, Inc.). RNA and DNA sequencing were performed on an Illumina HiSeq 2000 (PE125). For participants in the clinical study in Hong Kong, bacterial DNA and RNA were extracted from $200 \mathrm{mg}$ aliquots of frozen stool by Beijing Genome Institute (BGI). DNA was extracted using an E.Z.N.A. Stool DNA Kit according to the manufacturer's protocol. For RNA, rRNA was depleted using a Ribo-Zero ${ }^{\text {TM }}$ Magnetic Kit. The remaining total RNA was extracted using a RiboPure-Yeast Kit. All samples were sequenced on an Illumina HiSeq 4000 platform (Illumina, San Diego, California, USA; pairedend, insert size $350 \mathrm{bp}$, read length $150 \mathrm{bp}$ for DNA and 100 bp for RNA).

\section{Internal transcribed spacer sequencing}

All stool samples from both cohorts were processed by Novogene for internal transcribed spacer (ITS) sequencing. DNA was extracted using the following protocol: Stool samples were thoroughly mixed with $900 \mu \mathrm{L}$ of CTAB lysis buffer. All samples were incubated at $65^{\circ} \mathrm{C}$ for $60 \mathrm{~min}$ before being centrifuged at $12000 \times g$ for $5 \mathrm{~min}$ at $4{ }^{\circ} \mathrm{C}$. Supernatants were transferred to fresh $2-\mathrm{mL}$ microcentrifuge tubes and $900 \mu \mathrm{L}$ of phenol:chloroform: isoamyl alcohol (25:24:1, $\mathrm{pH}=6.7$; Sigma-Aldrich) was added for each extraction. Samples were mixed thoroughly prior to being incubated at room temperature for $10 \mathrm{~min}$. Phase separation occurred by centrifugation at $12,000 \times g$ for $15 \mathrm{~min}$ at $4{ }^{\circ} \mathrm{C}$, and the upper aqueous phase was re-extracted with a further $900 \mu \mathrm{L}$ of phenol: chloroform:isoamyl alcohol. Next, samples were centrifuged at $12,000 \times g$ for $10 \mathrm{~min}$ at $4{ }^{\circ} \mathrm{C}$, and the upper aqueous phases were transferred to fresh $2-\mathrm{mL}$ microcentrifuge tubes. The final extraction was performed with $900 \mu \mathrm{L}$ of chloroform:isoamyl alcohol (24:1), and layer separation occurred by centrifugation at $12,000 \times g$ for $15 \mathrm{~min}$ at $4{ }^{\circ} \mathrm{C}$. Precipitation of DNA was achieved by adding the upper phase from the last extraction step to $450 \mu \mathrm{L}$ of isopropanol (Sigma-Aldrich) containing $50 \mu \mathrm{L}$ of $7.5 \mathrm{M}$ ammonium acetate (Fisher). Samples were incubated at $-20{ }^{\circ} \mathrm{C}$ overnight, although shorter incubations $(1 \mathrm{~h})$ produced lower DNA yields. Samples were centrifuged at $7500 \times g$ for $10 \mathrm{~min}$ at $4{ }^{\circ} \mathrm{C}$, and supernatants were discarded. Finally, DNA pellets were washed three times in $1 \mathrm{~mL}$ of $70 \%(\mathrm{v} / \mathrm{v})$ ethanol (Fisher). The final pellet was air-dried and re-suspended in $200 \mu \mathrm{L}$ of $75 \mathrm{mM}$ TE buffer ( $\mathrm{pH}=8.0$; Sigma-Aldrich). The resulting fungal sequences were amplified using ITS2-F: 5' GCATCGATGAAGAACGCAGC-3' and ITS2-R: 5' TCCTCCGCTTATTGATATGC-3' primers $[59,60]$. ITS2 amplicons were generated in three steps by PCR with 38 cycles: $98^{\circ} \mathrm{C} 10$ s, $59^{\circ} \mathrm{C} 10$ s, and $72^{\circ} \mathrm{C} 30$ s followed by sequencing on the Illumina HiSeq platform $(2 \times 250 \mathrm{bp}$, Novogen, China).

\section{Metabolomics}

For 4 participants, bile acid profiles and MicrobioMET profiles were assessed by Metabo-Profile (Shanghai, China) using aliquots of frozen stool. For bile acid profiles, bile acid-free matrix (BAFM) was obtained using the charcoal-stripping protocol. Calibrators and quality controls were prepared for the BAFM and processed as for extraction of bile acids from stool samples. About 10 $\mathrm{mg}$ prechilled zirconium oxide beads were added to 10 mg stool with $15 \mu$ l ultrapure water. To each sample, a 
200- $\mu$ l aliquot of prechilled acetonitrile/methanol containing 10 internal standards was added for homogenization. After centrifugation at $13,500 \mathrm{rpm}$ and $4{ }^{\circ} \mathrm{C}$ for $20 \mathrm{~min}, 50 \mu \mathrm{l}$ supernatant was transferred to $96-$ well plates. Acetonitrile/water $(150 \mu \mathrm{l})$ was added for gentle shaking for $5 \mathrm{~min}$ before injection into an ultraperformance liquid chromatography column coupled to tandem mass spectrometry (UPLC-MS/MS) system to quantitate bile acids.

MicrobioMET profiles including aromatic phenols and indoles, phenolic acids, short-chain fatty acids and branched-chain amino acids, amino acids, and organic acids were quantitated using gas chromatography coupled to time-of-flight mass spectrometer (GCTOFMS). Stool aliquots $(50 \mathrm{mg})$ were homogenized with $300 \mu \mathrm{l} \mathrm{NaOH}(1 \mathrm{M})$ solution using a homogenizer and centrifuged at $13,500 \mathrm{rpm}$ and $4{ }^{\circ} \mathrm{C}$ for $20 \mathrm{~min}$. Supernatants $(200 \mu \mathrm{l})$ were transferred into autosampler vials and residue extracted with $200 \mu \mathrm{l}$ cold methanol. After a second homogenization and centrifugation, $167 \mu$ supernatant was combined with the first supernatant in the autosampler vial. Extracts were capped and used for automated sample derivatization by a robotic multipurpose sample MPS2 with dual heads (Gerstel, Muehlheim, Germany). Samples pre-treated with sodium sulfate were shaken at $1500 \mathrm{rpm}$ and $4{ }^{\circ} \mathrm{C}$ for $20 \mathrm{~min}$ and transferred to capped empty autosampler vials for the GC-TOFMS.

\section{Data processing}

\section{Quality control of sequence data}

Quality control of raw reads (DNA, RNA) used a previously described pipeline [61]. Adapter sequences, lowquality bases $(Q<20)$, duplicated reads, reads shorter than $75 \mathrm{bp}$ and reads mapping to the human genome with $95 \%$ coverage were filtered out. Computational scripts are at https://github.com/TingtZHENG/VirMiner/.

\section{In situ bacterial growth rate estimation}

Quality controlled FASTQ samples were sub-sampled to 2 million reads per sample. GRiD version 1.3 [25] was used with the corresponding stool database on subsampled samples to assess the growth bacterial strains. Default parameters were used but with minimum coverage threshold of 0.5 in order to investigate growth rates for different thresholds. After investigating the results, and as suggested by the GRiD authors, we continued with the growth estimates for strains with coverage 1.0 or higher. Statistical testing of (a) median growth rates and (b) the number of growing species was performed with a Wilcoxon signed-rank test. Normalized effect size $r$ was estimated using the $\mathrm{R}$ package "rcompanion" and its function "wilcoxonPairedR".

\section{Abundance profiling}

HUMAnN2 [19] version 0.11.1 was used to estimate gene family abundances in metagenomic DNA and RNA samples. Resulting reads per kilo-base (RPK) for gene family abundances at species level (including unclassified taxa) were further normalized by counts per million $(\mathrm{CPM})$, resulting in a transcripts per kilo-base million (TPKM) like normalization.

PIPITS pipeline [21] version 1.4.5 was used for ITS with default parameters including quality filtering, read-pair merging, ITS2 filtering, and chimaera removal. Remaining reads were binned based on $97 \%$ similarity as operational taxonomic unit and aligned to the UNITE fungi database using Mothur classifier [22]. For further downstream analysis, all samples were normalized by cumulative sum scaling using MetagenomeSeq [62].

For bile acid profiles, raw data from UPLC-MS/MS were processed using QuanMET software (v1.0, MetaboProfile) for peak integration, calibration and quantitation for each bile acid. The analyte concentration of unknown bile acid was calculated using a calibration curve.

For MicrobioMET profiles, raw data from the GCTOFMS were processed using proprietary software XploreMET (v2.0, Metabo-Profile) for automatic baseline denosing, smoothing, peak picking, and peak signal alignment. MS-based quantitative metabolomics determined the concentration of unknown metabolites by comparing the unknown to a calibration curve. Abundance of MirobioMET profiles was calculated to minimize large individual variations in metabolites.

\section{Metagenomic sequences from HUMAnN2 profiles}

TPKM-normalized gene family abundances from DNA were clustered using mgs-canopy version 1.0 software (https://anaconda.org/bioconda/mgs-canopy). We used standard parameters except for a Pearson correlation coefficient cut-off of 0.95 instead of the default 0.9. Gene family clusters with at least 700 genes were considered metagenomic sequences (MGS). Taxonomic annotation of MGS used species annotation information available for each gene family. We calculated contributions of each species to an MGS (including unclassified taxa). An MGS was annotated to species level using the largest gene family distribution if the gene contribution of that species was at least $51 \%$ and the second largest species (a) was "unclassified" or (b) contributed at most $10 \%$. MGS with more than $90 \%$ gene contribution from the same species were considered "pure" or "unambiguous". Using a more stringent species assignment than the original method [29], from a total of 213 MGS, we obtained 80 with species-level assignment (Suppl. Table 6). 


\section{Genomic strains from MGS}

MGS with species assignments were processed independently. Reads that (1) contributed to the abundance of an MGS, and (2) mapped to the inferred species (based on ChocoPhlAn reference [19]) were extracted. We used PanPhlAn [38] version 1.2.1.3 to create species-specific pangenomes based on reference genomes from the National Center for Biotechnology Information (Suppl. Table 14), mapped reads against the species pangenome, and calculated per-gene per-reference profiles. Gene abundance was normalized to reads per base. A gene was covered sufficiently if it had at least 0.5 reads per base. We accepted a strain reference if: (1) at least $90 \%$ of genes in the MGS were found to have sufficient coverage, and (2) the reference had the highest number of covered genes. For experimental verification, we considered using a commercially available strain if the number of covered genes was at most $1 \%$ less than the best-fitting strain.

\section{Diversity analysis}

Diversity analysis of fungal and bacterial communities was performed in $\mathrm{R}$ version 3.6.1 using the package vegan [63] version 2.5-5. Testing for significant differences in alpha diversity between time points was performed using a twosided Wilcoxon signed-rank test. Resulting $p$ values were adjusted for multiple testing using FDR. UniFrac metrics measured beta diversity by accounting for phylogenetic similarities of different species. Weighted UniFrac gives the most importance to dominant species. Unweighted UniFrac does not consider abundance. Generalized UniFrac with $\alpha=50 \%$ gives the most weight to moderately abundant species [64] and the generalized UniFrac with $\alpha$ $=75 \%$ to species with abundance between median and dominant levels.

\section{Transcriptional activity}

Relative abundances using DNA and RNA were normalized to transcripts per million. Let $f$ denote a gene or pathway. The transcriptional activity of $f$ is defined as the TPKM-normalized RNA abundance of $f$ divided by the TPKM-normalized DNA abundance of $f$.

\section{Core metatranscriptome}

The core metatranscriptome was described in [20]. Briefly, we used MetaCyc pathway relative abundances as generated by HUMANn2 for both DNA and RNA. We calculated transcriptional activity for each pathway. The core metatranscriptome was defined as the set of pathways with a sample prevalence of at least $80 \%$ with variable metatranscriptome having prevalence of 30 to $80 \%$. Pathways with less than $30 \%$ prevalence were ignored.

\section{Contributional alpha diversity}

We followed the procedure in [26], with some exceptions. For each MetaCyc pathway (PWY), the contribution of species to the pathway was determined. Ecological alpha diversity measures (Shannon and Simpson) were applied per sample and separately using DNA and RNA data. Mean diversity per sample was used to test for significant differences between time points using pairwise two-sided signed Wilcoxon tests. Resulting $p$ values were corrected for multiple testing using false discovery rate (FDR).

\section{Statistics for MGS and ITS abundance}

We used MetagenomeSeq [62] version 1.22.0 with a zero-inflated Gaussian mixture model. Following the MetagenomeSeq vignette, CSS normalization was applied on relative abundance data. All possible pairwise tests between the different sampling time points were performed (baseline, DT, EPT, and LPT time points). We controlled for patient-wise differences when possible. For MGS, D2 and D4 were excluded to improve signal quality. A $15 \%$ prevalence filter was used for each test independently. Controlling for multiple testing was performed on $p$ values using FDR.

\section{Two-way PERMANOVA testing}

Stool samples from the same participant were statistically dependent. To test for significant differences in means of beta diversity between different time points, two-way permutational analyses of variance (PERM ANOVA) were performed using "subject id" as covariate and "sample time point" as second independent variable. We performed tests on beta diversity matrices using the function "adonis" as implemented in R package vegan with 9999 permutations. We reported $F$ values, $R^{2}$, and $p$ values for "sample time point". $P$ values from pairwise PERMANOVA tests were corrected for multiple testing using FDR.

\section{Compositionality tests}

We implemented a compositionality test from Palleja et al. [27]. Briefly, we used the test to address the issue of false-positive and false-negative findings in compositional data [65]. We accepted significant findings for a species based on relative abundance only if they would still be significant if other species were removed from the abundance table. Therefore, if one species was removed, the data were total-sum normalized and $p$ values calculated. The procedure was repeated for all species. The final $p$ value for a species was determined using the highest calculated $p$ value. Thus, a species could not become significant because of depletion or inflation of another dominant species. Since this test was very 
conservative, we used a higher $q$ value of 0.1 to decide significance to avoid overlooking potential findings.

\section{Correlation analyses using stool metabolite abundance}

To identify metabolites with a potential effect on Candida, Saccharomyces, Penicillium, and Aspergillus spp., we calculated Spearman's correlations for total-sum scaling (TSS) ITS abundance and both bile acid and MicroMET profiles. To account for zero-inflation, we considered only samples with nonzero abundance of Candida albicans (5 samples). We then considered all significant correlations $(p<0.05)$ with an absolute correlation of at least $60 \%$.

To identify direct or indict bacterial producers of the metabolites, total-sum scaled MGS abundances were correlated with $\log 2$ transformed metabolites abundances. Correlation was inferred using sparse partial least squared analysis (sPLS) by utilizing relevance vectors ( $\mathrm{R}$ package mixOmics [30]).

\section{Co-abundance networks}

Co-abundance networks were created based on totalsum-normalized data using BAnOCC [31]. Significance of an edge was determined as described [20]. For posterior inference, we used the $95 \%$ credible interval. An edge was therefore considered significant if the corresponding 95\% credible interval did not contain zero. Only significant correlations with an absolute estimated coefficient of at least $30 \%$ were used for analysis. Significant changes in network structure between any two time points were determined using Wilcoxon signed-rank tests on node degree. Effect sizes are reported in terms of a standardized effect size analogous to the one used for the Mann-Whitney test, $r=z / \sqrt{n}$, where $z$ is the $z$ statistic of the paired test and $n$ is the number of observations. $r$ values are analogous to Pearson correlation coefficients. Hence, $r$ ranges from -1 (100\% decrease) to 1 (100\% increase). Formula and implementation can be found in the R package "rcompanion".

\section{Fungal species co-abundance network}

TSS-normalized operational taxonomic unit (OTU) abundances based on ITS2 data were used. OTUs detected in less than $10 \%$ of samples were removed. BAnOCC was executed with 5 chains, 5000 iterations, and 1000 warmup cycles to reach convergence. BAnOCC was used as described above.

\section{MGS-ITS network with BAnOCC}

MGS and ITS relative abundances were independently total-sum normalized. Only species measured in 25\% of samples were used further. Abundances of less prevalent species were summed per sample into a group called "other" to maintain library sizes. MGS and ITS features abundances were combined and analysed using BAnOCC as described above.

\section{RNA-PWY-ITS network with BAnOCC}

RNA abundances of PWY and ITS were independently total-sum normalized. A 50\% samples prevalence filter was applied to make this computation feasible and decrease false-positive rate. Abundances of less prevalent features were summed per sample into a group called "other". BAnOCC was used as described above.

\section{Supernatant experiments \\ Strains and culture conditions}

Odoribacter splanchnicus (DSM20712), Bacteroides eggerthii (DSM20697), C. albicans (SC5314/ ATCC MYA-2876), C. albicans (ATCC 10231), and C. albicans (ATCC 18804) were grown at $37^{\circ} \mathrm{C}$ under anaerobic conditions (anaerobic gas mixture, $95 \% \mathrm{~N}_{2}$, and $5 \% \mathrm{H}_{2}$ ) in pre-reduced modified Gifu anaerobic media (mGAM; Nissui Pharmaceutical Co. Ltd.) broth for liquid cultures or mGAM broth supplemented with agar (Nissui Pharmaceutical Co. Ltd.) for growth on plates.

\section{Sterile bacterial supernatants}

Bacterial strains grown for $48 \mathrm{~h}$ in mGAM broth were subcultured 1:50 in fresh mGAM broth and grown for $48 \mathrm{~h}$ in anaerobic conditions at $37^{\circ} \mathrm{C}$. Bacterial cultures were spun down at $11,000 \times g$ for $5 \mathrm{~min}$. Supernatants were carefully removed and filtered through $0.2-\mu \mathrm{M}$ syringe filters to remove bacteria in suspension.

\section{Supernatant growth inhibition assays}

C. albicans growth rates were analysed in $200 \mu$ liquid mGAM with $50 \%$ or $100 \%$ sterile bacterial supernatant added. C. albicans inoculations were at 1:1000 from an overnight culture grown in aerobic conditions at $37^{\circ} \mathrm{C}$. Cultures were in 96-well microtiter plates at $37^{\circ} \mathrm{C}$ with orbital shaking $365 \mathrm{cpm}(2 \mathrm{~mm})$. Cell densities were measured every $10 \mathrm{~min}$ at optical density $600 \mathrm{~nm}$ (OD600) using a microtiter reader (BioTek ELx800). Growth rates were calculated by plotting the log OD measurements in log phase and calculating slopes for timepoints in log phase where $r^{2}$ was closest to 1 , using at least 12 time points ( $2 \mathrm{~h}$ apart).

\section{Supernatant metabolite assays}

Analysis of SCFA in samples was carried out by MSOmics as follows. Samples were acidified using hydrochloride acid, and deuterium labelled internal standards where added. All samples were analysed in a randomized order. Analysis was performed using a high polarity column (Zebron ${ }^{\mathrm{m} \mathrm{m}}$ ZB-FFAP, GC Cap. Column $30 \mathrm{~m} \times 0.25$ $\mathrm{mm} \times 0.25 \mu \mathrm{m})$ installed in a GC (7890B, Agilent) coupled with a quadropole detector (5977B, Agilent). 
The system was controlled by ChemStation (Agilent). Raw data was converted to netCDF format using Chemstation (Agilent), before the data was imported and processed in Matlab R2014b (Mathworks, Inc.) using the PARADISe software described by Johnsen et. al [68].

Other compounds such as bile acids were analysed using MS/MS. The analysis was carried out using a Thermo Scientific Vanquish LC coupled to Thermo Q Exactive HF MS. An electrospray ionization interface was used as ionization source. Analysis was performed in negative and positive ionization mode. The UPLC was performed using a slightly modified version of the protocol described by Catalin et al. (UPLC/MS Monitoring of Water-Soluble Vitamin Bs in Cell Culture Media in Minutes, Water Application note 2011, 720004042en). Peak areas were extracted using Compound Discoverer 2.0 (Thermo Scientific). Identification of compounds were performed at four levels: level 1-identification by retention times (compared against in-house authentic standards), accurate mass (with an accepted deviation of 3 ppm), and MS/MS spectra; level 2a-identification by retention times (compared against in-house authentic standards), accurate mass (with an accepted deviation of $3 \mathrm{ppm}$ ); level $2 \mathrm{~b}$-identification by accurate mass (with an accepted deviation of $3 \mathrm{ppm}$ ), and MS/MS spectra; level 3-identification by accurate mass alone (with an accepted deviation of $3 \mathrm{ppm})$.

\section{C. albicans growth inhibition by metabolites}

Metabolites were acquired from the companies SigmaAldrich, Merck KGaA, and Roth. More specific details can be found in Suppl. Table 15.

\section{C. albicans growth curves}

Dilution series of metabolites in water were started at concentrations approximately 10 -fold below maximum solubility in water where applicable (Suppl. Table 16). Dilutions were in synthetic SD medium ( $1 \times$ yeast nitrogen base, $2 \%$ glucose, $\left.0.5 \% \mathrm{NH}_{4} \mathrm{SO}_{4}\right)$. C. albicans was grown overnight in YPD (1\% yeast extract, $2 \%$ peptone, $2 \%$ glucose), washed $3 \times$ in sterile water, and inoculated at $1: 100\left(\mathrm{OD}_{600} \approx 0.2\right)$. Absorbance was measured every 15 min with an infinite M200pro microwell plate reader (Tecan, Austria) set to $30^{\circ} \mathrm{C}$ with intermittent shaking (10 s orbital shaking before each measurement). Generation times were calculated from the obtained triplicate growth curves.

\section{Host cell damage assays}

To determine the influence of metabolites on the general capacity of $C$. albicans to cause host cell damage, we used an established epithelial cell model based on the vaginal epithelial cell line A431. A431 were grown in
RPMI media containing 10\% foetal bovine serum (FBS), and $200 \mu \mathrm{l}$ cells at $10^{5}$ cells $/ \mathrm{ml}$ were seeded into 96-well plates and incubated at $37^{\circ} \mathrm{C}, 5 \% \mathrm{CO}_{2}$. After $48 \mathrm{~h}$, cells were washed with $1 \times \mathrm{PBS}$, and $100 \mu \mathrm{l}$ compound at indicated concentrations was added, followed by $100 \mu \mathrm{l} \mathrm{Can-}$ dida cells at multiplicity of infection 1. Incubation continued under the same conditions for $24 \mathrm{~h}$. Basal lactate dehydrogenase (LDH) release (low control) was determined with $200 \mu \mathrm{l}$ RPMI, and maximum LDH release (high control) determined by addition of $100 \mu \mathrm{l} 0.5 \%$ Triton X-100 to cells in $100 \mu \mathrm{l}$ RPMI. Plate were centrifuged at $250 \times g$ for $10 \mathrm{~min}$ and supernatants were removed and diluted 1:10 and mixed with $100 \mu$ l freshly prepared LDH assay mix (Roche). After $25 \mathrm{~min}$ at room temperature in the dark, LDH activity was determined with a microplate reader (Tecan infinite M200) as absorbance (A) at $492 \mathrm{~nm}$, with $660 \mathrm{~nm}$ as a reference. Damage was calculated as $\left(A_{\text {sample }}-A_{\text {low }}\right) /\left(A_{\text {high }}-A_{\text {low }}\right)$.

\section{C. albicans morphology}

The effect of metabolites on C. albicans morphology was tested at all concentrations used in cell damage assays. Metabolites were diluted in $250 \mu \mathrm{l}$ RPMI medium with $10 \% \mathrm{FBS}$ and added to $250 \mu \mathrm{l} \mathrm{C}$. albicans in RPMI in 24-well plates to indicated concentrations. Plates were incubated at $37^{\circ} \mathrm{C}$ and $5 \% \mathrm{CO}_{2}$ for $4 \mathrm{~h}$ to induce hyphae formation. Medium was removed and cells fixed with Histofix $4 \%$ formaldehyde solution. Morphology was evaluated using an inverse microscope (Axio Zeiss Vert. A1) to differentiate yeasts, hyphae, and pseudohyphae.

\section{Pairwise co-cultivation experiments}

Interactions between $C$. albicans and B. eggerthii and $O$. splanchnicus were assayed via pairwise cultivations. $C$. albicans cell counts were compared to control conditions of cultivation without bacteria.

Fungal and bacterial cells were grown anaerobically at $37^{\circ} \mathrm{C}$ for up to $48 \mathrm{~h}$ in mGAM and used as inocula for pairwise experiments. Inocula biomasses were estimated via OD600 and adjusted to 1.0 by diluting in appropriate media. Inocula were transferred to microplates containing the same media to a final OD600 of 0.01. Ratios of fungal to bacteria cells were 1:1. Microplates were incubated at $37^{\circ} \mathrm{C}$ statically under anaerobic conditions. Cell counts from inocula were resolved, prior to the cocultivation experiments, via flow cytometry (BD LSRFortessa, BD Biosciences, Franklin Lakes, NJ, USA).

Five microplates were prepared using the same inoculum. Microplates were removed from the anaerobic chamber every $5 \mathrm{~h}(0,5,10,15$, and $20 \mathrm{~h}$ cultivation). Cells were immediately fixed in $2 \%$ formaldehyde for 15 min at room temperature by mixing an equal amount of sample volume and 4\% formaldehyde (Sigma-Aldrich, Saint Louis, MI, USA) [66, 67]. After fixing, total $C$. 
albicans cells were counted via flow cytometry (BD LSRFortessa, BD Biosciences, Franklin Lakes, NJ, USA). Experiments were performed in triplicate.

\section{Supplementary information}

Supplementary information accompanies this paper at https://doi.org/10. 1186/s40168-020-00899-6.

\section{Additional file 1: Supplementary Figures. \\ Additional file 2. Statistical results for differences in fungal relative} abundance between time points at genus level.

Additional file 3. Statistical results for differences in fungal relative abundance between time points at species level.

Additional file 4. Fungal network properties and statistical results for differences in node degree between time points.

Additional file 5. Growth rate indices of bacterial strains per sample as estimated by GRiD. GRiD values of 1 imply no growth.

Additional file 6. Statistical results for differences between groupcentroids between different time points using distance-based redundancy analysis.

Additional file 7. Information on taxonomic annotation of co-abundant gene clusters (CAG). For each CAG, the bacterial species contributing the highest-and second highest-number of genes is shown. For the most contributing species, the percentage of genes assigned to that species is shown in "PercentageOfTaxonContribution". The column "trusted" indicates if the corresponding cluster passed our annotation criteria.

Additional file 8. Statistical results for differences in MGS relative abundance between time points.

Additional file 9. MGS network properties and statistical results for differences in node degree between time points.

Additional file 10. Cross-kingdom correlations representing the MGSITS correlation network. Correlations are listed for each time point.

Additional file 11. List of bacterial species with predicted positive or negative correlation to selected fungal species. The table further contains information on the corresponding MGS, time-related fold-changes, and for which antibiotic drugs the observation was made.

Additional file 12. Statistical results for differences in node degree between time points in RNA-MetaCyc network. Results are given for the entire network as well as differences based on funcational categories.

Additional file 13. Significant correlations between fungal species and stool metabolites.

Additional file 14. Patient information.

Additional file 15. List of published genome assemblies available on NCBI. A subset of these were used to infer strain information on several MGS clusters.

Additional file 16. Metabolite company information.

Additional file 17. Dilution series of metabolites for which we tested their effect on Candida albicans growth, morphology, and host cell damage.

\section{Acknowledgements}

Not applicable.

\section{Authors' contributions}

Conceptualizations: M.O.A.S. and G.P. Funding acquisitions: M.O.A.S. and G.P. Investigations: R.V.U., F.L., P.C.Y.W., K.-F.C., C.-C.T., R.P.K.L., S.S., J.C., S.B., A.M., and B.S. Methodology: B.S., J.C., R.V.U., A.M., R.S., F.L., S.B., D.L., and K.K. Project administration: G.P. Resources: G.P., B.H., M.O.A.S., and P.C.Y.W. Supervision: G.P. Validation: all authors. Visualization: B.S., J.C., and R.S.. Writing-original draft: B.S. and G.P. Writing —review and editing: B.S, M.O.A.S., B.H., and G.P. The authors read and approved the final manuscript.

\section{Funding}

G.P. and B.H. would like to thank the Deutsche Forschungsgemeinschaft (DFG) CRC/Transregio 124 "Pathogenic fungi and their human host: Networks of interaction", subprojects B5, C1 and INF. M.O.A.S. would like to thank the Novo Nordisk Foundation under NFF grant number NNF10CC1016517, The Novo Nordisk Foundation, Challenge programme, CaMiT under grant agreement NNF17CO0028232, and The Lundbeck Foundation under grant agreement R140-2013-13496. The Hong Kong study (P.C.Y.W.) was partly funded by the Collaborative Innovation Center for Diagnosis and Treatment of Infectious Diseases, Ministry of Education, China. Open access funding provided by Projekt DEAL.

\section{Availability of data and materials}

Metagenomic and transcriptomic data generated and analysed during the current study are available in the NCBI SRA repository as Bio Projects PRJNA573821, PRJNA573905, and PRJNA579284. Data from previous work analysed in the current study is available in the NCBI SRA repository as Bio Project PRJINA588313.

Metabolite profiles were uploaded to MetaboLights (https://www.ebi.ac.uk/ metabolights/) with accession MTBLS1846.

Code used for non-standard statistical tests are available as a git repository on https://bitbucket.org/Xentrics/antibiotic-gut-fungi/src/master/.

\section{Ethics approval and consent to participate}

The Danish study was approved by the local ethics committee in Region Zealand, Denmark (SJ-383). The Hong Kong study was approved by the Institutional Review Board of The University of Hong Kong/Hospital Authority Hong Kong West Cluster (UW 17-042).

\section{Consent for publication}

Not applicable.

\section{Competing interests}

The authors declare that they have not competing interests.

\section{Author details}

'Leibniz Institute for Natural Product Research and Infection Biology_-Systems Biology and Bioinformatics, Hans Knöll Institute, Adolf-Reichwein-Straße 23, 07745 Jena, Germany. ${ }^{2}$ Department of Medicine, State Key Laboratory of Pharmaceutical Biotechnology, The University of Hong Kong, Hong Kong, SAR, China. ${ }^{3}$ Leibniz Institute for Natural Product Research and Infection Biology-Microbial Pathogenicity Mechanisms, Hans Knöll Institute, Adolf-Reichwein-Straße 23, 07745 Jena, Germany. ${ }^{4}$ Novo Nordisk Foundation Center for Biosustainability, Technical University of Denmark, Kemitorvet 220, DK-2800 Lyngby, Denmark. ${ }^{5}$ Department of Microbiology, Li Ka Shing Faculty of Medicine, The University of Hong Kong, Pokfulam, Hong Kong. ${ }^{6}$ Emergency Medicine Unit, Li Ka Shing Faculty of Medicine, The University of Hong Kong, Pokfulam, Hong Kong. ${ }^{7}$ State Key Laboratory of Emerging Infectious Diseases, The University of Hong Kong, Pokfulam, Hong Kong. ${ }^{8}$ Collaborative Innovation Center for Diagnosis and Treatment of Infectious Diseases, The University of Hong Kong, Pokfulam, Hong Kong.

Received: 15 May 2020 Accepted: 24 July 2020

Published online: 12 September 2020

References

1. Turnbaugh PJ, Ley RE, Hamady M, Fraser-Liggett CM, Knight R, Gordon JI. The Human Microbiome Project. Nature [Internet]. 2007 [cited 2019 May 10];449:804-10. Available from: http://www.nature.com/articles/nature06244

2. Shreiner $A B$, Kao JY, Young VB. The gut microbiome in health and in disease. Curr Opin Gastroenterol [Internet]. NIH Public Access; 2015 [cited 2019 May 16];31:69-75. Available from: http://www.ncbi.nlm.nih.gov/ pubmed/25394236.

3. Chakraborty A, Ghosh S, Chowdhary G, Maulik U, Chakrabarti S. DBETH: A database of bacterial exotoxins for human. Nucleic Acids Res [Internet]. Narnia; 2012 [cited 2019 May 10];40:D615-20. Available from: https:// academic.oup.com/nar/article-lookup/doi/10.1093/nar/gkr942. 
4. Forster SC, Browne HP, NK. HPMCD: the database of human microbial communities from metagenomic datasets and microbial reference genomes. Nucleic Acids Res. 2016.

5. Iliev ID, Funari VA, Taylor KD, Nguyen Q, Reyes CN, Strom SP, et al Interactions between commensal fungi and the C-type lectin receptor Dectin-1 influence colitis. Science (80- ). American Association for the Advancement of Science; 2012;336:1314-7.

6. Wheeler ML, Limon JJ, Bar AS, Leal CA, Gargus M, Tang J, et al. Immunological consequences of intestinal fungal dysbiosis. Cell Host MicrobeElsevier. 2016;19:865-73.

7. Li X, Leonardi I, Semon A, Doron I, Gao IH, Putzel GG, et al. Response to fungal dysbiosis by gut-resident CX3CR1+ mononuclear phagocytes aggravates allergic airway disease. Cell Host Microbe [Internet]. Cell Press; 2018 [cited 2019 Sep 4];24:847-856.e4. Available from: https://www. sciencedirect.com/science/article/abs/pii/S1931312818305602.

8. Kim Y-G, Udayanga KGS, Totsuka N, Weinberg JB, Núñez G, Shibuya A. Gut dysbiosis promotes M2 macrophage polarization and allergic airway inflammation via fungiinduced PGE2. Cell Host MicrobeElsevier. 2014;15:95-102.

9. Dethlefsen $\mathrm{L}$, Relman DA. Incomplete recovery and individualized responses of the human distal gut microbiota to repeated antibiotic perturbation. Proc Natl Acad Sci U S A [Internet]. National Academy of Sciences; 2011 [cited 2019 May 10];108 Suppl 1:4554-61. Available from: http://www.ncbi. nlm.nih.gov/pubmed/20847294

10. Noverr MC, Noggle RM, Toews GB, Huffnagle GB. Role of antibiotics and fungal microbiota in driving pulmonary allergic responses. Infect Immun Am Soc Microbiol. 2004;72:4996-5003.

11. Palleja A, Mikkelsen KH, Forslund SK, Kashani A, Allin KH, Nielsen T, et al. Recovery of gut microbiota of healthy adults following antibiotic exposure. Nat MicrobiolNature Publishing Group. 2018;1.

12. Dollive $S$, Chen $Y-Y$, Grunberg S, Bittinger $K$, Hoffmann C, Vandivier $L$, et al, Fungi of the murine gut: episodic variation and proliferation during antibiotic treatment. PLoS One [Internet]. Public Library of Science; 2013 [cited 2019 May 16];8:e71806. Available from: http://www.ncbi.nlm.nih.gov/ pubmed/23977147.

13. Cabral DJ, Penumutchu S, Norris C, Morones-Ramirez JR, Belenky P. Microbial competition between Escherichia coli and Candida albicans reveals a soluble fungicidal factor. Microb Cell. Shared Science Publishers; 2018;5:249.

14. Peleg AY, Hogan DA, Mylonakis E. Medically important bacterial-fungal interactions. Nat Rev Microbiol [Internet]. Nature Publishing Group; 2010 [cited 2019 May 10];8:340-9. Available from: http://www.nature.com/articles/ nrmicro2313.

15. Frey-Klett P, Burlinson P, Deveau A, Barret M, Tarkka M, Sarniguet A. Bacterial-fungal interactions: hyphens between agricultural, clinical, environmental, and food microbiologists. Microbiol Mol Biol Rev [Internet] American Society for Microbiology; 2011 [cited 2019 May 10];75:583-609. Available from: http://www.ncbi.nlm.nih.gov/pubmed/22126995, http:// www.pubmedcentral.nih.gov/articlerender.fcgi?artid=PMC3232736.

16. Huttenhower C, Gevers D, Knight R, Abubucker S, Badger JH, Chinwalla AT, et al. Structure, function and diversity of the healthy human microbiome. Nature. 2012;486:207-14

17. Segata N, Waldron L, Ballarini A, Narasimhan V, Jousson O, Huttenhower C. Metagenomic microbial community profiling using unique clade-specific marker genes. Nat Methods [Internet]. Nature Publishing Group; 2012 [cited 2019 May 27];9:811-4. Available from: http://www.nature.com/articles/ nmeth.2066

18. Truong DT, Tett A, Pasolli E, Huttenhower C, Segata N. Microbial strain-level population structure and genetic diversity from metagenomes. Genome Res [Internet]. Cold Spring Harbor Laboratory Press; 2017 [cited 2019 May 10];27: 626-38. Available from: http://www.ncbi.nlm.nih.gov/pubmed/28167665.

19. Franzosa EA, Mclver $L$, Rahnavard $G$, Thompson LR, Schirmer $M$, Weingart $\mathrm{G}$, et al. Species-level functional profiling of metagenomes and metatranscriptomes. Nat MethodsNature Publishing Group. 2018;15:962.

20. Abu-Ali GS, Mehta RS, Lloyd-Price J, Mallick H, Branck T, Ivey KL, et al. Metatranscriptome of human faecal microbial communities in a cohort of adult men. Nat MicrobiolNature Publishing Group. 2018;3:356.

21. Gweon HS, Oliver A, Taylor J, Booth T, Gibbs M, Read DS, et al. PIPITS: an automated pipeline for analyses of fungal internal transcribed spacer sequences from the Illumina sequencing platform. Methods Ecol EvolWiley Online Library. 2015;6:973-80.

22. Schloss PD, Westcott SL, Ryabin T, Hall JR, Hartmann M, Hollister EB, et al. Introducing mothur: open-source, platform-independent, community- supported software for describing and comparing microbial communities. Appl Environ Microbiol [Internet]. American Society for Microbiology; 2009 [cited 2019 May 13];75:7537-41. Available from: http://www.ncbi.nlm.nih. gov/pubmed/19801464.

23. Aykut B, Pushalkar S, Chen R, Li Q, Abengozar R, Kim Jl, et al. The fungal mycobiome promotes pancreatic oncogenesis via activation of MBL. Nature [Internet]. Nature Publishing Group; 2019 [cited 2019 Nov 4];574:264-7. Available from: http://www.nature.com/articles/s41586-019-1608-2.

24. Qin J, Li R, Raes J, Arumugam M, Burgdorf KS, Manichanh C, et al. A human gut microbial gene catalogue established by metagenomic sequencing. NatureNature Publishing Group. 2010;464:59-65.

25. Emiola A, Oh J. High throughput in situ metagenomic measurement of bacterial replication at ultra-low sequencing coverage. Nat Commun [Internet]. Springer US; 2018;9. Available from: http://dx.doi.org/https://doi. org/10.1038/s41467-018-07240-8.

26. Schirmer M, Franzosa EA, Lloyd-Price J, Mclver LJ, Schwager R, Poon TW, et al. Dynamics of metatranscription in the inflammatory bowel disease gut microbiome. Nat MicrobiolNature Publishing Group. 2018;3:337.

27. Palleja A, Kashani A, Allin KH, Nielsen T, Zhang C, Li Y, et al. Roux-en-Y gastric bypass surgery of morbidly obese patients induces swift and persistent changes of the individual gut microbiota. Genome Med [Internet]. 2016;8:67. Available from: https://doi.org/https://doi.org/10.1186/ s13073-016-0312-1.

28. Dehoux P, Marvaud JC, Abouelleil A, Earl AM, Lambert T, Dauga C. Comparative genomics of Clostridium bolteae and Clostridium clostridioforme reveals species-specific genomic properties and numerous putative antibiotic resistance determinants. BMC Genomics [Internet]. BioMed Central; 2016 [cited 2019 Sep 23];17:819. Available from: http:// bmcgenomics.biomedcentral.com/articles/10.1186/s12864-016-3152-x.

29. Nielsen HB, Almeida M, Juncker AS, Rasmussen S, Li J, Sunagawa S, et al. Identification and assembly of genomes and genetic elements in complex metagenomic samples without using reference genomes. Nat BiotechnolNature Publishing Group. 2014;32:822.

30. Pedersen HK, Forslund SK, Gudmundsdottir V, Petersen A $\varnothing$, Hildebrand F, Hyötyläinen T, et al. A computational framework to integrate high-throughput '-omics' datasets for the identification of potential mechanistic links. Nat Protoc [Internet]. Nature Publishing Group; 2018 [cited 2019 May 17];13:2781-800. Available from: http://www.nature.com/articles/s41596-018-0064-z.

31. Schwager E, Mallick H, Ventz S, Huttenhower C. A Bayesian method for detecting pairwise associations in compositional data. PLoS Comput BiolPublic Library of Science. 2017;13:e1005852.

32. Tamanai-Shacoori Z, Smida I, Bousarghin L, Loreal O, Meuric V, Fong SB, et al. Roseburia spp.: a marker of health? Future Microbiol. Future Medicine Ltd.; 2017. p. 157-170.

33. Machiels K, Joossens M, Sabino J, De Preter V, Arijs I, Eeckhaut V, et al. A decrease of the butyrate-producing species roseburia hominis and faecalibacterium prausnitzii defines dysbiosis in patients with ulcerative colitis. GutBMJ Publishing Group. 2014;63:1275-83.

34. Martínez I, Lattimer JM, Hubach $\mathrm{KL}$, Case JA, Yang J, Weber CG, et al. Gut microbiome composition is linked to whole grain-induced immunological improvements. ISME JNature Publishing Group. 2013;7:269-80.

35. Shibuya A, Shibuya K. Exploring the gut fungi-lung allergy axis. Cell Host MicrobeElsevier. 2018;24:755-7.

36. Costea Pl, Munch R, Coelho LP, Paoli L, Sunagawa S, Bork P. metaSNV: A tool for metagenomic strain level analysis. Wang K, editor. PLoS One [Internet]. Public Library of Science; 2017 [cited 2019 May 10];12:e0182392. Available from: https://dx.plos.org/https://doi.org/10.1371/journal.pone. 0182392.

37. Luo C, Knight R, Siljander H, Knip M, Xavier RJ, Gevers D. ConStrains identifies microbial strains in metagenomic datasets. Nat Biotechnol [Internet]. Nature Publishing Group; 2015 [cited 2019 May 10];33:1045-52. Available from: http://www.nature.com/articles/nbt.3319.

38. Albanese D, Donati C. Strain profiling and epidemiology of bacterial species from metagenomic sequencing. Nat CommunNature Publishing Group. 2017:8:2260

39. Nguyen LN, Lopes LCL, Cordero RJB, Nosanchuk JD. Sodium butyrate inhibits pathogenic yeast growth and enhances the functions of macrophages. J Antimicrob Chemother. 2011;66:2573-80.

40. Mason KL, Downward JRE, Falkowski NR, Young VB, Kao JY, Huffnagle GB. Interplay between the gastric bacterial microbiota and Candida albicans during postantibiotic recolonization and gastritis. Infect Immun. 2012;80:150-8. 
41. Zhang L, Bahl MI, Roager HM, Fonvig CE, Hellgren LI, Frandsen HL, et al. Environmental spread of microbes impacts the development of metabolic phenotypes in mice transplanted with microbial communities from humans. ISME JNature Publishing Group. 2017;11:676-90.

42. Staley C, Kaiser T, Beura LK, Hamilton MJ, Weingarden AR, Bobr A, et al. Stable engraftment of human microbiota into mice with a single oral gavage following antibiotic conditioning. MicrobiomeBioMed Central. 2017;5:87.

43. Fouladi F, Glenny EM, Matthew ECB, Sioda M, Thomas SA, Wang Y, et al. Sequence variant analysis reveals poor correlations in microbial taxonomic abundance between humans and mice after gnotobiotic transfer. ISME J [Internet]. Springer US; 2020; Available from: http://dx.doi.org/https://doi. org/10.1038/s41396-020-0645-z.

44. Sovran B, Planchais J, Jegou S, Straube M, Lamas B, Natividad JM, et al. Enterobacteriaceae are essential for the modulation of colitis severity by fungi. Microbiome [Internet]. BioMed Central Ltd.; y [cited 2020 Apr 14];6: 152. Available from: https://microbiomejournal.biomedcentral.com/articles/1 0.1186/s40168-018-0538-9.

45. World Health Organization (WHO). World health organization model list of essential medicines. Ment Holist Heal Some Int Perspect [Internet]. 2019; 119-34. Available from: https://www.who.int/medicines/publications/ essentialmedicines/en/.

46. Maier L, Goemans CV, Pruteanu M, Wirbel J, Kuhn M, Cacace E, et al. Dissecting the collateral damage of antibiotics on gut microbes. bioRxivCold Spring Harbor Laboratory. 2020;2020(01):09.893560.

47. Vandeputte D, Kathagen G, D'Hoe K, Vieira-Silva S, Valles-Colomer M, Sabino J, et al. Quantitative microbiome profiling links gut community variation to microbial load. Nature [Internet]. Nature Publishing Group; 2017 [cited 2020 Apr 21];551:507-11. Available from: http://dx.doi.org/https://doi.org/10.1038/ nature24460.

48. Roager HM, Hansen LBS, Bahl MI, Frandsen HL, Carvalho V, Gøbel RJ, et al. Colonic transit time is related to bacterial metabolism and mucosal turnover in the gut. Nat MicrobiolNature Publishing Group. 2016;1:1-9.

49. Gosalbes MJ, Durbán A, Pignatelli M, Abellan JJ, Jiménez-Hernández N, Pérez-Cobas AE, et al. Metatranscriptomic approach to analyze the functional human gut microbiota. PLoS OnePublic Library of Science. 2011; 6:e17447.

50. Guinan J, Villa P, Thangamani S. Secondary bile acids inhibit Candida albicans growth and morphogenesis. Pathog Dis Oxford University Press. 2018;76.

51. Guinan J, Wang S, Hazbun TR, Yadav H, Thangamani S. Antibiotic-induced decreases in the levels of microbial-derived short-chain fatty acids correlate with increased gastrointestinal colonization of Candida albicans. Sci Rep [Internet]. Nature Publishing Group; 2019 [cited 2019 Oct 23];9:8872. Available from: http://www.nature.com/articles/s41598-019-45467-7.

52. Witchley JN, Penumetcha P, Abon N V., Woolford CA, Mitchell AP, Noble SM. Candida albicans morphogenesis programs control the balance between gut commensalism and invasive infection. Cell Host Microbe [Internet]. Cell Press; 2019 [cited 2019 Mar 22];25:432-443.e6. Available from: https://www.sciencedirect.com/science/article/pii/S1931312819301040.

53. Louis P, Hold GL, Flint HJ. The gut microbiota, bacterial metabolites and colorectal cancer. Nat Rev Microbiol [Internet]. Nature Publishing Group; 2014 [cited 2019 Jul 2];12:661-72. Available from: http://www.nature.com/ articles/nrmicro3344.

54. Morrison DJ, Preston T. Formation of short chain fatty acids by the gut microbiota and their impact on human metabolism. Gut Microbes. Taylor and Francis Inc.; 2016. p. 189-200.

55. Pompella A, Corti A. Editorial: the changing faces of glutathione, a cellular protagonist. Front Pharmacol [Internet]. Frontiers; 2015 [cited 2019 Aug 9];6: 98. Available from: http://www.frontiersin.org/Experimental_Pharmacology_ and_Drug_Discovery/10.3389/fphar.2015.00098/full.

56. Zhang Y, Cai C, Yang Y, Weng L, Wang L. Blocking of Candida albicans biofilm formation by cis-2-dodecenoic acid and trans-2-dodecenoic acid. J Med Microbiol [Internet]. Microbiology Society; 2011 [cited 2019 Jul 2];60: 1643-50. Available from: http://jmm.microbiologyresearch.org/content/ journal/jmm/10.1099/jmm.0.029058-0.

57. Yang $\mathrm{DL}$, Zhang $Y Q$, Hu YL, Weng LX, Zeng GS, Wang LH. Protective effects of cis-2-dodecenoic acid in an experimental mouse model of vaginal candidiasis. Biomed Environ Sci [Internet]. Biomedical and Environmental Sciences; 2018 [cited 2019 Jul 2];31:816-28. Available from: https://www. sciencedirect.com/science/article/pii/S0895398819300534
58. Raymond F, Ouameur AA, Déraspe M, lqbal N, Gingras H, Dridi B, et al. The initial state of the human gut microbiome determines its reshaping by antibiotics. ISME JNature Publishing Group. 2016;10:707-20.

59. White TJ, Bruns T, Lee S, Taylor J. Amplification and direct sequencing of fungal ribosomal RNA genes for phylogenetics. In: Innis MA, Gelfand DH, Sninsky JJ, White TJ, editors. PCR protocols: a guide to methods and applications. PCR Protoc a Guid to methods Appl. San Diego, CA: Academic Press; 1990. p. 315-22.

60. Zuo T, Wong SH, Cheung CP, Lam K, Lui R, Cheung K, et al. Gut fungal dysbiosis correlates with reduced efficacy of fecal microbiota transplantation in Clostridium difficile infection. Nat Commun [Internet]. Nature Publishing Group; 2018 [cited 2019 Aug 5];9:3663. Available from: http://www.nature.com/articles/s41467-018-06103-6.

61. Kang K, Ni Y, Li J, Imamovic L, Sarkar C, Kobler MD, et al. The environmental exposures and inner- and intercity traffic flows of the metro system may contribute to the skin microbiome and resistome. Cell Rep [Internet]. Cell Press; 2018 [cited 2019 May 21];24:1190-1202.e5. Available from: https:// www.sciencedirect.com/science/article/pii/S2211124718310519.

62. Paulson JN, Stine OC, Bravo HC, Pop M. Differential abundance analysis for microbial marker-gene surveys. Nat Methods Nature Publishing Group. 2013; 10:1200.

63. Dixon P. VEGAN, a package of $R$ functions for community ecology. J Veg SciWiley Online Library. 2003;14:927-30.

64. Chen J, Bittinger K, Charlson ES, Hoffmann C, Lewis J, Wu GD, et al. Associating microbiome composition with environmental covariates using generalized UniFrac distances. BioinformaticsOxford University Press. 2012; 28:2106-13.

65. Knight R, Vrbanac A, Taylor BC, Aksenov A, Callewaert C, Debelius J, et al. Best practices for analysing microbiomes. Nat Rev MicrobiolNature Publishing Group. 2018;1.

66. Chao Y, Zhang T. Optimization of fixation methods for observation of bacterial cell morphology and surface ultrastructures by atomic force microscopy. Appl Microbiol BiotechnolSpringer. 2011;92:381-92.

67. Moloney M, McDonnell L, O'Shea H. Atomic force microscopy of BHK-21 cells: an investigation of cell fixation techniques. UltramicroscopyNorthHolland. 2004;100:153-61.

68. Lea G. Johnsen, Peter B. Skou, Bekzod Khakimov, Rasmus Bro. Gas chromatography - mass spectrometry data processing made easy. J Chromatogr A. 2017;1503:57-64.

\section{Publisher's Note}

Springer Nature remains neutral with regard to jurisdictional claims in published maps and institutional affiliations.

Ready to submit your research? Choose BMC and benefit from:

- fast, convenient online submission

- thorough peer review by experienced researchers in your field

- rapid publication on acceptance

- support for research data, including large and complex data types

- gold Open Access which fosters wider collaboration and increased citations

- maximum visibility for your research: over $100 \mathrm{M}$ website views per year

At $\mathrm{BMC}$, research is always in progress.

Learn more biomedcentral.com/submissions 